\title{
VOF simulations of the contact angle dynamics during the drop spreading: standard models and a new wetting force model.
}

\author{
Ilias Malgarinos ${ }^{1}$, Nikolaos Nikolopoulos ${ }^{1,5}$, Marco Marengo ${ }^{2,4}$, Carlo Antonini ${ }^{2,3}$ and Manolis Gavaises ${ }^{1}$ \\ 1: School of Engineering and Mathematical Sciences, City University London, Northampton Square, EC1V \\ OHB London, UK, *Corresp. Author - e-mail: Ilias.Malgarinos.1@city.ac.uk \\ 2: Department of Engineering, University of Bergamo, viale Marconi 5, 24044 Dalmine, Italy \\ 3: Laboratory of Thermodynamics in Emerging Technologies, Department of Mechanical and Process \\ Engineering, ETH Zurich, Zurich 8092, Switzerland \\ 4: School of Computing, Engineering and Mathematics, University of Brighton, Brighton BN2 4GJ, UK \\ 5: Centre for Research and Technology Hellas, Chemical Process and Energy Resources Institute, \\ Egialeias 52, Marousi, Athens, Gr-15125, Greece
}

\begin{abstract}
A novel numerical implementation for the adhesion of liquid droplets impacting normally on solid dry surfaces is presented; the physical model is implemented as a source term in the momentum equation of a Navier-Stokes CFD flow solver as an "adhesion-like" force which acts at the triple-phase contact line as a result of capillary interactions between the liquid drop and the solid substrate. The advantage of this new approach, compared to the majority of existing models, is that the dynamic contact angle forming during the surface wetting process is not inserted as a boundary condition, but is derived implicitly by the induced fluid flow characteristics (interface shape) and the adhesion physics of the gasliquid-surface interface (triple line), starting only from the advancing and receding equilibrium contact angles. These angles are required in order to define the wetting properties of liquid phases when interacting with a solid surface. The numerical simulations capture the liquid-air interface movement by considering the Volume of Fluid (VOF) method and utilizing an automatic local grid refinement technique in order to increase the accuracy of the predictions at the area of interest, and simultaneously minimize numerical diffusion of the interface. The proposed model is validated against previously reported experimental data of normal impingement of water droplets on dry surfaces at room temperature. A wide range of impact velocities, i.e. Weber numbers from as low as 0.2 up to 117, both for hydrophilic $\left(\theta_{\mathrm{adv}}=10^{\circ}-70^{\circ}\right)$ and hydrophobic $\left(\theta_{\mathrm{adv}}=105^{\circ}-120^{\circ}\right)$ surfaces, has been examined. Predictions include in addition to droplet spreading dynamics, the estimation of the dynamic contact angle; the latter is found in reasonable agreement against available experimental measurements. It is thus concluded that implementation of this model is an effective approach for overcoming the need of a pre-defined dynamic contact angle law, frequently adopted as an approximate boundary condition for such simulations. Clearly, this model is mostly influential during the spreading phase for the cases of low
\end{abstract}


We number impacts (We $<~ 80$ ) since for high impact velocities, inertia dominates significantly over capillary forces in the initial phase of spreading.

Keywords: CFD; VOF; dynamic contact angle; droplet impingement; dynamic grid refinement

\begin{tabular}{|c|c|c|c|c|c|}
\hline \multicolumn{3}{|c|}{ Nomenclature } & \multirow[b]{2}{*}{ Greek letters } & & \\
\hline Symbol & Quantity & units & & & \\
\hline $\mathrm{Ca}$ & Capillary Number & $(-)$ & $\alpha$ & $\begin{array}{l}\text { Liquid volume } \\
\text { fraction }\end{array}$ & \\
\hline$C_{p}$ & Pressure Coeff. & $(-)$ & & $\begin{array}{l}\text { interfacial } \\
\text { tension }\end{array}$ & $\mathrm{N} / \mathrm{m}$ \\
\hline D & Droplet Diameter & $\mathrm{m}$ & $\Delta \mathrm{x}$ & cell width & $\mathrm{m}$ \\
\hline$f_{a d h} / f_{W F M}$ & Adhesion Force & $\mathrm{N}$ & $\Rightarrow$ & & \\
\hline$\vec{f}_{W F M}^{v}$ & $\begin{array}{l}\text { Adhesion Stress } \\
\text { term (volumetric) }\end{array}$ & $\mathrm{N} / \mathrm{m}^{3}$ & $\Delta \theta$ & $\begin{array}{l}\text { Contact angle } \\
\text { hysteresis }\end{array}$ & $\left({ }^{\circ}\right)$ \\
\hline$f_{H}$ & $\begin{array}{l}\text { Hoffman's } \\
\text { function }\end{array}$ & & $\theta$ & contact angle & $\left({ }^{\circ}\right)$ \\
\hline $\overrightarrow{\mathrm{f}}_{\sigma}$ & Surface Tension & $\mathrm{N} / \mathrm{m}^{3}$ & K & curvature & $1 / \mathrm{m}$ \\
\hline $\overrightarrow{\mathrm{g}}$ & $\begin{array}{l}\text { Gravitational } \\
\text { acceleration }\end{array}$ & $\mathrm{m} / \mathrm{s}^{2}$ & $\mu$ & $\begin{array}{l}\text { dynamic } \\
\text { viscosity }\end{array}$ & $\mathrm{kg} / \mathrm{ms}$ \\
\hline K & $\begin{array}{l}\text { Proportionality } \\
\text { constant }\end{array}$ & $(-)$ & $\rho$ & density & $\mathrm{kg} / \mathrm{m}^{3}$ \\
\hline I & $\begin{array}{l}\text { Length of contact } \\
\text { line }\end{array}$ & $\mathrm{m}$ & $\sigma$ & $\begin{array}{l}\text { surface tension } \\
\text { coefficient }\end{array}$ & $\mathrm{N} / \mathrm{m}$ \\
\hline L & $\begin{array}{l}\text { Total Length of } \\
\text { contact line }\end{array}$ & $\mathrm{m}$ & $\tau$ & $\begin{array}{l}\text { non- } \\
\text { dimensional } \\
\text { time }\end{array}$ & $(-)=t u / D$ \\
\hline$\hat{n}$ & $\begin{array}{l}\text { Free-surface } \\
\text { normal }\end{array}$ & $(-)$ & Subscripts & & \\
\hline$\vec{n}_{b}$ & $\begin{array}{l}\text { Boundary face } \\
\text { vector looking } \\
\text { outside }\end{array}$ & $(-)$ & $\infty$ & $\begin{array}{l}\text { corresponds to } \\
\text { freestream } \\
\text { conditions }\end{array}$ & \\
\hline$P$ & Pressure & $\mathrm{Pa}$ & $a d v$ & advancing & \\
\hline r & $\begin{array}{l}\text { rim axisymmetric } \\
\text { distance }\end{array}$ & $\mathrm{m}$ & dyn & dynamic & \\
\hline $\mathrm{R}$ & Droplet Radius & $\mathrm{m}$ & eq & equilibrium & \\
\hline $\mathrm{Re}=\rho_{\text {liq }} \mathrm{U}_{0} \mathrm{D}_{0} / \mu_{\text {liq }}$ & Reynolds number & $(-)$ & gas & gas & \\
\hline $\mathrm{t}$ & time & $\mathrm{s}$ & liq & liquid & \\
\hline$\vec{T}$ & Stress tensor & $\mathrm{kg} / \mathrm{ms}^{2}$ & $\max$ & maximum & \\
\hline u & velocity & $\mathrm{m} / \mathrm{s}$ & 0 & initial condition & \\
\hline $\mathrm{V}_{\text {cell }}$ & Cell volume & $\mathrm{m}^{3}$ & rec & receding & \\
\hline
\end{tabular}




\begin{tabular}{|l|l|l|l|l|}
\hline We $=\rho_{\text {liq }} U_{0}^{2} D_{0} / \sigma$ & Weber number & $(-)$ & \\
\hline $\mathrm{X}$ & x-axis distance & $\mathrm{m}$ & & \\
\hline $\mathrm{Y}$ & X-axis distance & $\mathrm{m}$ & & \\
\hline
\end{tabular}

\section{Introduction}

The impact of liquid droplets onto solid surfaces is a very interesting physical process that takes place in many engineering applications. For example, cooling of hot surfaces (e.g. electronic circuits) can be achieved by impingement of cold liquid droplets onto them [1]; in the work of Kandlikar et al. [2], a comprehensive review of jet impingement and spray cooling works is presented, focusing on the maximum heat removal value which can be achieved (reaching up to $1000 \mathrm{~W} / \mathrm{cm}^{2}$ ). Another interesting application of droplet impingement dynamics is in internal combustion engines, where the interaction of the atomized fuel droplets with the piston and liner walls play an important role on combustion characteristics and fluid mixing inside the cylinder [3]; the so-called $1^{\text {st }}$ generation direct injection gasoline engines [4] utilized this process in order to direct the injected liquid towards the spark plug and create the required stratification within the engine cylinder. In a very different sector, that of spray coatings, droplets impinge on a solid surface and solidify, to form a coating layer so that the surface can acquire specific properties (wear resistant, protection against corrosion, desirable electrical conductivity) [5]. In aviation applications, water supercooled droplets formed in clouds can freeze after impact on the surface of airfoils, resulting in the formation of an ice layer $[6,7]$, which can have detrimental effect on the functionality of wings, blades and engines during flight. More recently, new superhydrophobic selfcleaning materials are introduced and applied especially in photovoltaic panels, allowing the raindrops to slide down on the glass surfaces carrying the dust, thus promoting the achievement of higher absorption rates of radiation [8] of such surfaces.

Droplet dynamics and their interaction with solid surfaces have fascinated researchers from the time of 1805 (Thomas Young [9]) and 1867 (Worthington [10]). Since then, a vast number of studies have been published that deal with the underlying physics both theoretically and experimentally; various numerical models implemented in Computational Fluid Dynamics (CFD) codes are also capable of predicting the underlying physical mechanisms. The readers can find a comprehensive review on the phenomenon of drop impact onto a solid surface, from the flow dynamics point of view, in the works of Rein [11], Yarin [12] and Marengo et al. [13]. In the study of Yarin [12], the expected outcomes of this process are identified. More specifically, droplet impingement onto a flat solid surface can lead to "deposition", "rebound" or "splashing". These studies also suggest that the most crucial dimensionless parameters, which govern this phenomenon, are the Reynolds and Weber numbers.

In the scope of theoretical works, some researchers focus on the complex dynamics governing the three phase (liquid-gas-solid) contact line [14, 15], while an issue arise due to the hydrodynamic singularity at the three phase line [16]. In other theoretical approaches, the droplet maximum spreading is estimated based on an energy conservation approach, in terms of initial kinetic and surface energy before impact and surface energy along with viscous dissipation at its maximum spreading. In 1991, Chandra and Avedisian [17] using such an approach reached to a derivation of maximum spread for impact of a 
droplet on a hot flat surface, while afterwards, in 1996, Pasandideh et al. [18] provided an estimation of the spreading factor for a cold surface, taking into consideration the existence of a viscous boundary layer in which most of the kinetic energy is dissipating. This new energy conservation formula resulted in the following equation:

$$
\frac{D_{\max }}{D_{0}}=\sqrt{\frac{W e+12}{3(1-\cos \theta)+4(W e / \sqrt{\operatorname{Re}})}}
$$

which takes into account along with the effect of Reynolds and Weber numbers, the angle the gas-liquid interface makes with the solid substrate at the end of the advancing phase, for the estimation of the maximum spreading factor. Similar equations can be found in the works of Mao [19], Ukiwe et al. [20], Vadillo et al. [21], but all of them follow the same underlying main principles as those initially proposed by Pasandideh et al. [18]. Other analytical works assume that the deformed shape of the droplet, when it spreads, is the same with that of a truncated sphere [22] or a cylindrical disk [23].

Concerning experimental measurements, the results of a vast number of relevant campaigns $[19,21,22$, 24-37] can be found in literature, where the effect of basic parameters such as droplet basic properties (viscosity, density, surface tension), droplet size, impact velocity, solid surface roughness and wettability on droplet spreading are investigated explicitly. More recently, the interest has been turned towards superhydrophobic and complex surfaces $[13,38]$, as there is a great challenge in studying the behavior of droplets coming in contact with such substrates. One common realisation resulting from the aforementioned investigations is the appearance of the wetting contact angle, namely the advancing and the receding ones, as one of the most influential parameters during droplet spreading onto solid substrates [7]. These have been measured in some cases as a function of substrate manufacture properties, i.e. smooth/rough glass in Rioboo et al. [30], or even substrate roughness amplitude [26, 38]. However, as it has been proved from very early times (Jiang et al. [39] in 1979), the dynamic contact angle of the rim varies considerably, especially during the advancing phase of droplet spreading.

More recently, due to the great advancements in high-speed photography, the dynamic contact angle was investigated more explicitly. In fact, Hung et al. [37], using a "droplet impingement imaging system" managed to digitize the shape of the droplet at each time instant during the spreading process extrapolating considerable information about its variation as function of the rim velocity. Still, only limited experimental works depicting this change of contact angle values during the spreading period can be found. To the best of authors' knowledge only five works can be tracked [18, 21, 36, 40, 41]. For example, the work of Bayer et al. [36] reported a significant dynamic contact angle variation during the hysteresis period from a starting value of $125^{\circ}$ down to of $40^{\circ}$ till the start of the receding phase. It is therefore clear that the definition of static angles is not enough to fully define the complex physics at the three-phase contact line formed during droplet impact. Indeed, the advancing and receding contact angles are usually measured during quasi-steady conditions, whereas the process of droplet impingement is highly dynamic and instationary. As a consequence, it is important for CFD simulations to account for this dynamic condition; models capable of predicting this instantaneous dynamic contact angle seem to be more appealing and, in contrast to most of the current CFD approaches that adopt a pre-defined contact angle, inserted as a boundary condition. 
Focusing to CFD works, a large number of numerical studies dealing with the impingement of a single liquid droplet onto a flat solid surface have been reported. Selective (but not limited) studies include different models for interface tracking, i.e. Lagrangian (Marker cell [42], deforming grid [43, 44], Front tracking [45, 46], LCRM [47]), Eulerian (SOLA-VOF [18, 48, 49], Volume of Fluid Method (VOF) [50-53], CLSVOF [54], Level Set [55, 56], VOF+PLIC [57], PLIC, VOF with Young reconstruction [41, 58]), EulerianLagrangian [59, 60], Lattice-Boltzmann [61, 62], and lately the phase field approach (Cahn-Hillard equation) [63]. Other studies also account for the effect of heat transfer mechanisms [44, 48, 49, 52, 53, 64-67] between the impinging droplet and the substrates but these fall outside the scope of the present study.

Undoubtedly, one of the most challenging tasks of a CFD model is the correct prediction of the gas-liquid interaction on a three-phase contact line, while simultaneously trying to avoid numerical diffusion. A very interesting review on computational methods used for the prediction/simulation of moving contact line phenomena is presented in the work of Sui et al. [68]. The main modeling approach followed in literature is to insert the contact angle as a boundary condition at the active boundary cells, lying in contact with the wall. Indeed, the only variation among all the methods followed so far is upon how this insertion is applied, rather than predicting it from first principles. Fukai et al. [43] were the first to apply the contact angle as a boundary condition in cases of water droplet impingement onto a cold flat surface following a 2-D axisymmetric Lagrangian approach in a grid, which was deformed according to the shape of the droplet free surface. In that approach, the mean curvature of the free surface next to the wall was adjusted so that the desired pre-defined contact angle was reached. Other studies follow the basic approach proposed by Brackbill et al. [69], i.e. the widely adopted CSF model (selectively [41, 50, 57-59]). According to that, the normal to the free surface unit vector on the wall cell is adjusted according to the pre-defined contact angle. This influences the surface tension force, which is inserted in the NavierStokes momentum equation, resulting in the "intentional" turning of the rim according to the prescribed contact angle value. Pasandideh et al. [18] made the same adjustment for a SOLA-VOF code, so did Ganesan and Sashikumaar [60] for a Lagrangian-Eulerian approach. Similar methods were applied in subsequent works. Other approaches follow either the extrapolation of the free surface outside of the wall, so that the free surface normal to the wall can be adjusted, and used afterwards in the reconstruction process $[47,54]$. In [61] where the Lattice-Boltzmann method was used, an external force field was introduced at the active cells being in contact with the wall. Finally, in the Level Set function, utilized in the work of Caveziel et al. [55], a different re-initialization method near the wall was adopted, while Griebel et al. [56] used a fixed value Neumann boundary condition at the wall for the free surface normal of the Level Set function. Table 1 summarizes the approaches used and the range of conditions of the aforementioned representative studies. Only previously simulated cases concerning water droplets have been included, as the present work pays attention on low viscosity liquids. Additionally, due to the large surface tension coefficient of air/water, the induced surface tension force is quite high relevant to viscous ones, and the resulting net of forces at the triple contact line highly dominated by surface tension effects. For that reason, the correct simulation of water droplet impingement cases, to the authors' opinion, serves as the most challenging conditions for the validation of the proposed model. Additionally, there is a lack of simulated conditions for water droplet impingement, especially onto hydrophilic surfaces and for low and medium Weber numbers. 


\begin{tabular}{|c|c|c|c|c|c|c|c|c|}
\hline Year & Simulation & $\boldsymbol{O}_{\mathrm{adv}}\left({ }^{\circ}\right)$ & We & $\operatorname{Re}$ & Model & Contact angle model & Velocity Calc & Experiments \\
\hline 1995 & $\begin{array}{l}\text { Fukai et } \\
\text { al.[43] }\end{array}$ & $49-92$ & $\begin{array}{l}112- \\
733\end{array}$ & $\begin{array}{l}5476- \\
14062\end{array}$ & $\begin{array}{l}\text { 2-D } \\
\text { axisymmetric } \\
\text { Lagrangian } \\
\text { deforming } \\
\text { grid }\end{array}$ & Constant angle (Adv-Rec) & $\begin{array}{l}\text { Marker } \\
\text { velocity }\end{array}$ & Own \\
\hline 1996 & $\begin{array}{l}\text { Pasandideh } \\
\text { et al. [18] }\end{array}$ & 110 & 27 & 2000 & $\begin{array}{l}\text { axisymmetric } \\
\text { modified } \\
\text { SOLA - VOF }\end{array}$ & $\begin{array}{l}\text { Constant equilibrium } \\
\text { angle/Dynamic from } \\
\text { experiment }\end{array}$ & $\mathrm{N} / \mathrm{A}$ & Own \\
\hline 2000 & $\begin{array}{l}\text { Bussmann et } \\
\text { al. [58] }\end{array}$ & 110 & 440 & 8000 & $\begin{array}{l}\text { piecewise } \\
\text { linear volume } \\
\text { tracking } \\
\text { method }\end{array}$ & $\begin{array}{l}\text { Dynamic - linear in relation to } \\
\text { velocity }\end{array}$ & $\begin{array}{l}\text { cell center } \\
\text { velocity }\end{array}$ & Own \\
\hline 2003 & $\begin{array}{l}\text { Francois et } \\
\text { al. [59] }\end{array}$ & 87.4 & 30 & 2772 & $\begin{array}{l}\text { immersed } \\
\text { boundary } \\
\text { method } \\
\text { Eulerian- } \\
\text { Lagrangian }\end{array}$ & $\begin{array}{l}\text { Constant equilibrium angle } \\
\text { /Dynamic linear in relation to } \\
\text { velocity }\end{array}$ & $\begin{array}{l}\text { 2nd marker } \\
\text { point veloc } \\
\left(0.8^{*} \text { grid }\right. \\
\text { spacing) }\end{array}$ & $\begin{array}{c}\text { Kim and Chun } \\
\text { [22] }\end{array}$ \\
\hline 2005 & $\begin{array}{l}\text { Gunjal et al. } \\
\text { [57] }\end{array}$ & $35-110$ & $3-567$ & $\begin{array}{c}924- \\
10000\end{array}$ & $\begin{array}{l}\text { VOF - } \\
\text { piecewise } \\
\text { linear }\end{array}$ & $\begin{array}{l}\text { Stepwise time variation } \\
\text { according to experiments } \\
\text { (every } 15 \text { steps) }\end{array}$ & $\mathrm{N} / \mathrm{A}$ & Own \\
\hline 2005 & $\begin{array}{l}\text { Sikalo et al. } \\
\quad[50]\end{array}$ & 105 & 92 & 4018 & $\begin{array}{l}\text { VOF-based } \\
\text { free-surface } \\
\text { capturing } \\
\text { method }\end{array}$ & Kistler's law & $\begin{array}{l}\text { differentiation } \\
\text { of the wetted } \\
\text { radius }\end{array}$ & Own \\
\hline 2007 & $\begin{array}{l}\text { Mukherjee et } \\
\text { al. [61] }\end{array}$ & 87.4 & 30 & 2772 & LBM & Kistler's law & $\begin{array}{l}\text { differentiation } \\
\text { of the wetted } \\
\text { radius }\end{array}$ & $\begin{array}{c}\text { Kim and Chun } \\
{[22]}\end{array}$ \\
\hline 2008 & $\begin{array}{l}\text { Caviezel et } \\
\text { al. [55] }\end{array}$ & 100 & 53 & 3245 & Level Set & Constant equilibrium angle & N/A & $\begin{array}{c}\text { Rioboo et al. } \\
\text { [30] }\end{array}$ \\
\hline 2008 & $\begin{array}{l}\text { Roisman et } \\
\text { al. [41] }\end{array}$ & 120 & $\begin{array}{c}0.88- \\
7.9\end{array}$ & D- & $\begin{array}{l}\text { VOF with } \\
\text { Youngs } \\
\text { interface } \\
\text { tracking }\end{array}$ & Kistler's law & $\begin{array}{l}\text { algebraic } \\
\text { system of } \\
\text { equations }\end{array}$ & Own \\
\hline 2009 & $\begin{array}{l}\text { Yokoi et al. } \\
\quad[54]\end{array}$ & 107 & 32 & 2 & CLSVOF & $\begin{array}{l}\text { Modified Tanner's law/ } \\
\text { Constant angle (Adv-Rec) }\end{array}$ & $\begin{array}{l}\text { u parallel to } \\
\text { wall closest to } \\
\text { the contact } \\
\text { point } x\end{array}$ & own \\
\hline 2009 & $\begin{array}{l}\text { Shin et al. } \\
\text { [47] }\end{array}$ & 92 & 129 & 5878 & $\begin{array}{l}\text { level contour } \\
\text { reconstruction } \\
\text { method }\end{array}$ & $\begin{array}{c}\text { if } \theta>\theta a d v, \theta=\theta a d v, \text { if } \theta<\theta \text { rec, } \\
\theta=\theta \text { rec, else } \theta=\theta\end{array}$ & $\begin{array}{l}\text { shear strain } \\
\text { rate(du/dn) } \\
\quad * \Delta x\end{array}$ & $\begin{array}{l}\text { Fukai et al. } \\
\text { [43] }\end{array}$ \\
\hline 2012 & $\begin{array}{l}\text { Ganesan et } \\
\text { al. [60] }\end{array}$ & 105 & & 4018 & $\begin{array}{l}\text { Lagrangian- } \\
\text { Eulerian } \\
\text { approach } \\
\text { (ALE-FEM) }\end{array}$ & $\begin{array}{l}\text { Constant equilibrium angle } \\
\text { /Hocking's model/Jiang's } \\
\text { modle/Bracke's model }\end{array}$ & Lagrangian & $\begin{array}{l}\text { Sikalo et al. } \\
\quad[50]\end{array}$ \\
\hline 2013 & $\begin{array}{c}\text { Griebel et al. } \\
{[56]}\end{array}$ & 107 & 32 & 22800 & Level Set & $\begin{array}{l}\text { Yokoi's model/Shikhmurzaev's } \\
\text { model }\end{array}$ & $\begin{array}{l}\text { u parallel to } \\
\text { wall closest to } \\
\text { the contact } \\
\text { point } x\end{array}$ & $\begin{array}{l}\text { Yokoi et al. } \\
\quad[54]\end{array}$ \\
\hline
\end{tabular}

Table 1. Contact angle models and velocity calculation methods used in simulations from literature for water droplet impingement.

In Table 1, Fukai et al. [43] simulated impingement onto hydrophilic surfaces for a range of We numbers between 112 and 733. In other works, Ganesan et al. [60] simulated case \#7 of Sikalo et al. (Table 2) which concerns the impingement of a water droplet onto a slightly hydrophobic wax surface $\left(\theta_{\text {adv }}=\right.$ $105^{\circ}$ ) and afterwards presented their simulations for a droplet impact characterized by a very low contact angle $\left(\theta_{\mathrm{adv}}=10^{\circ}\right)$, without a comparison against experimental data. Gunjal et al. [57] simulated the impingement of a water droplet on a glass surface using stepwise time variation of contact angles according to the experimental values, so that they can simulate the temporal evolution observed during the experiment, in a validated way. Even if this approach seems to be a promising one, especially for the case of droplet impingement onto hydrophilic cases, where the temporal variation of the contact angle is quite intensive, the simulation always needs the previous knowledge of the experimental data for each impact condition. Bussmann et al. [58] observed this contact angle variation for a stainless steel 
surface. Vadillo et al. [21] showed that during the first milliseconds of a water droplet impingement onto two hydrophilic surfaces with equilibrium contact angles of $5^{\circ}$ and $50^{\circ}$ respectively, the dynamic contact angle can reach values of approximately up to $80^{\circ}-90^{\circ}$. The dynamic contact angle models presented in Table 1 are representative of what has been documented in literature. For a more thorough, interesting and recent review of the available dynamic contact angle models, the readers can found information in the work by Saha and Mitra [70]. However, the question of how to simulate a priori a nonexperimentally investigated case, for which contact angle data are not available, still remains to be answered.

Efforts towards the development of more complete models that can predict the dynamic change of contact angle during the droplet impact have also been reported. Roisman et al. [41] stressed out that the use of a dynamic contact angle model is very important in a CFD simulation, so that the temporal evolution of the phenomenon can be captured more accurately. Yokoi et al. [54] showed that in order to achieve a very good agreement between experimental data and simulation results, especially for the recoiling phase, the right use of a contact angle model is a necessity. In all the previously mentioned cases, the introduction of the contact angle between the droplet and the solid surface is achieved by specifying the contact angle (static or dynamic) as a boundary condition, i.e. as an input for the numerical simulation. This implementation has the basic drawback, that the dynamic contact angle is not a result of the simulated underlying physical mechanisms, and the induced fluid flow characteristics, but is dependent on the previously known values of contact angle temporal variation and the right calculation of the rim velocity.

In CFD simulations, especially using the VOF method, the extrapolation of the contact line velocity, when the interface of the droplet covers 2 or 3 cells, is not an easy process. Therefore, a new model needs to be developed that can dynamically alter the contact angle based on the induced fluid flow characteristics, rather than insert it as a boundary condition.

That is the basic aim of this study, i.e. to develop a new model that does not prescribe the contact angle (or its temporal evolution), but lets the net of forces acting on the free surface of the droplet (capillary, surface tension) and the 3-phase contact line to determine the liquid/gas interface interaction with the surface.

\section{Wettability}

\subsection{Dynamic contact angle models}

Most dynamic contact angle models relate contact line velocity to the dynamic contact angle, which is inserted as a boundary condition. The dynamic contact angle models are briefly presented here below.

\subsubsection{Quasi-dynamic contact angle mode (Advancing - Receding)}

In the simplest possible advancing-receding contact angle model, a given fixed value for both the advancing and the receding contact angles is imposed as a boundary condition when the contact line spreads or recoils. This model has been used widely in literature, and can be found selectively in the 
works of Pasandideh et al. [49] and Berberovic et al. [71]. The basic approach is simply represented by the two equations:

$\theta_{\text {dyn }}=\theta_{\text {adv }}$ if $\mathrm{u}_{\text {conline }} \geq 0$

$\theta_{\text {dyn }}=\theta_{\text {rec }}$ if $\mathrm{u}_{\text {conline }}<0$

\subsubsection{Kistler's law}

In the work of Kistler [72], the dynamic contact angle is given as a function of the contact line velocity through the Capillary number and the inverse of Hoffman's function:

$$
\begin{aligned}
& \theta_{d y n}=f_{H}\left[\operatorname{Ca}+f_{H}^{-1}\left(\theta_{e q}\right)\right] \\
& f_{H}=\arccos \left\{1-2 \tanh \left[5.16\left(\frac{x}{1+1.31 x^{0.99}}\right)\right]^{0.706}\right\}^{\prime}\left(x=C a+f_{H}^{-1}\left(\theta_{e q}\right)\right) \\
& . C a=\frac{\mu_{\text {liq }} u_{\text {conline }}}{\sigma}
\end{aligned}
$$

\subsubsection{Shikhmurzaev's model}

Shikhmurzaev [73] gave a relation for the dynamic contact angle, as a function of several parameters, i.e.:

$$
\begin{aligned}
& \cos \theta_{d y n}=\cos \theta_{e q}-\frac{2 u^{*}\left(a_{1}+a_{2} u_{0}^{*}\right)}{\left(1-a_{2}\right)\left[\left(a_{1}+u^{* 2}\right)^{1 / 2}+u^{*}\right]} \\
& u^{*}=a_{3} \frac{\mu_{\text {liq }} u_{\text {conline }}}{\sigma}, u_{0}^{*}=\frac{\sin \theta_{d y n}-\theta_{d y n} \cos \theta_{d y n}}{\sin \theta_{d y n} \cos \theta_{d y n}-\theta_{d y n}}, a_{1}=1+\left(1-a_{2}\right)\left(\cos \theta_{e q}-a_{4}\right)
\end{aligned}
$$

where $a_{2}=0.54, a_{3}=12.5, a_{4}=0.07$ are phenomenological constants (values taken from [70]).

Basically, the dynamic contact angle models of Kistler and Shikhmurzaev try to deal with the difference observed in experiments between the dynamic contact angle (at microscopic length scale) and the apparent contact angle (at macroscopic length scale) within the inner region near the triple line point. A wide range of empirical correlations can be found in literature (a comprehensive review is presented in the work of Saha and Mitra [70]), that relate the contact line velocity with the dynamic contact angle in order to account for this inner region. This is also addressed in the works of Sikalo et al. [74] and Roisman et al. [41]. The differences in dimensionless spreading dynamics parameters (e.g. temporal evolution of spreading ratio, maximum spreading) which are observed between the simple quasidynamic model and these models may be related to that blurred distinction (Yokoi et al. [54]). 


\subsection{Wetting force model (WFM)}

The idea behind the derivation of the Wetting Force Model comes from the work of Antonini et al. [75]. The authors investigated experimentally the adhesion force of a droplet sliding down an inclined surface and they derived a methodology for the prediction of this "adhesion" force. The "adhesion force" acts at the contact line, and is responsible for drop capillary adhesion to the surface. In their work [75], this adhesion force, for a contact line of arbitrary shape, is given by:

$f_{\text {adh }}=-\sigma \int_{0}^{L} \cos \theta(I) \cos \psi(I) d l$

where $L$ is the total length of the contact line, as visualized in Figure 1 (from [75]). For the validation of their methodology, the magnitude of the "adhesion" force was explicitly calculated, from the component of gravitational force (Figure 1a) acting on the droplet parallel to the solid surface, at the exact moment when it starts moving and for this specific angle of inclination.

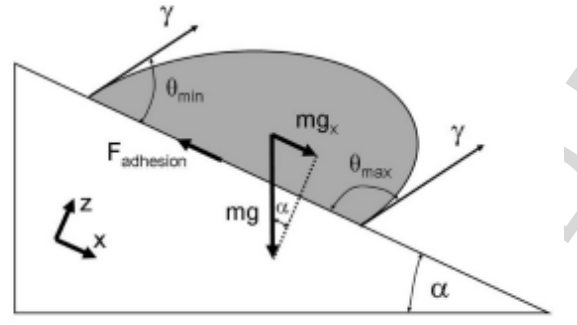

a

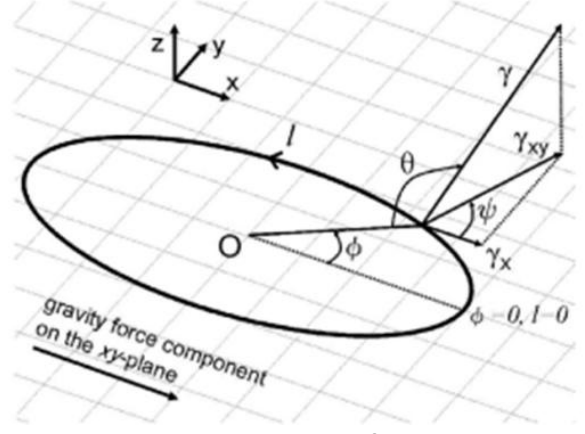

b

Figure 1. Adhesion force. a) Droplet sliding down an incline. At the moment it starts moving, $F_{\text {adhesion }}=m g \sin a$. b) Projection of the contact area of the droplet on the incline. Both Images taken from Antonini et al. [75]

Since the exact shape of the contact line and the complete contact angle distribution may be unknown, a simplified approach is typically used in many works, e.g. by Extrand and Kumagai [76], to estimate the capillary adhesion force. They refer to the adhesion force for solid/liquid/vapor systems, as being mathematically described by the following equation, both for static conditions, as well as dynamic conditions:

$\frac{f_{a d h}}{r}=K \sigma\left(\cos \theta_{r e c}-\cos \theta_{a d v}\right)$

where $\mathrm{K}$ is a fitting factor. In fact, the limit of this equation is the presence of the fitting factor $\mathrm{K}$, which changes by changing the liquid-solid system.

Both the previously mentioned cases (Antonini et al. [75] and Extrand and Kumagai [76]) refer to static conditions (a droplet at rest onto a solid surface on the verge of starting to move), but a more complete model should be as well checked for its validation not only under stationary and/or quasi-steady conditions, but as well under dynamic ones. In this light, the scope of this study is to check the modelling 
of an adhesion force model to the drop impact problem. The new model proposed considers for the effect that this additional (except from the standard interfacial tensions) contact line force will have - in relation to the "adhesion" force on the inclined surface or the one referred to in eq. 10 - on a spreading rate of a liquid droplet impacting onto both hydrophilic or hydrophobic surfaces. This additional term as implemented here describes the adhesion type force assumed to be parallel to the wall, as in Figure 2, and is expressed as:

$$
\mathrm{d} f_{W F M}=\sigma\left(\cos \theta_{e q}-\cos \theta_{d y n}\right) \mathrm{d} l=\sigma\left(\cos \theta_{e q}-\cos \theta_{d y n}\right) r d \phi
$$

Where the equivalence $\mathrm{d} l=r \mathrm{~d} \phi$ holds for a circular contact line, which is coherent with assumptions of axisymmetric flow for 2-D simulations. The direction of this stress term is presented in Figure 2 and is linked to the "adhesion" force reported before (eq. 10).

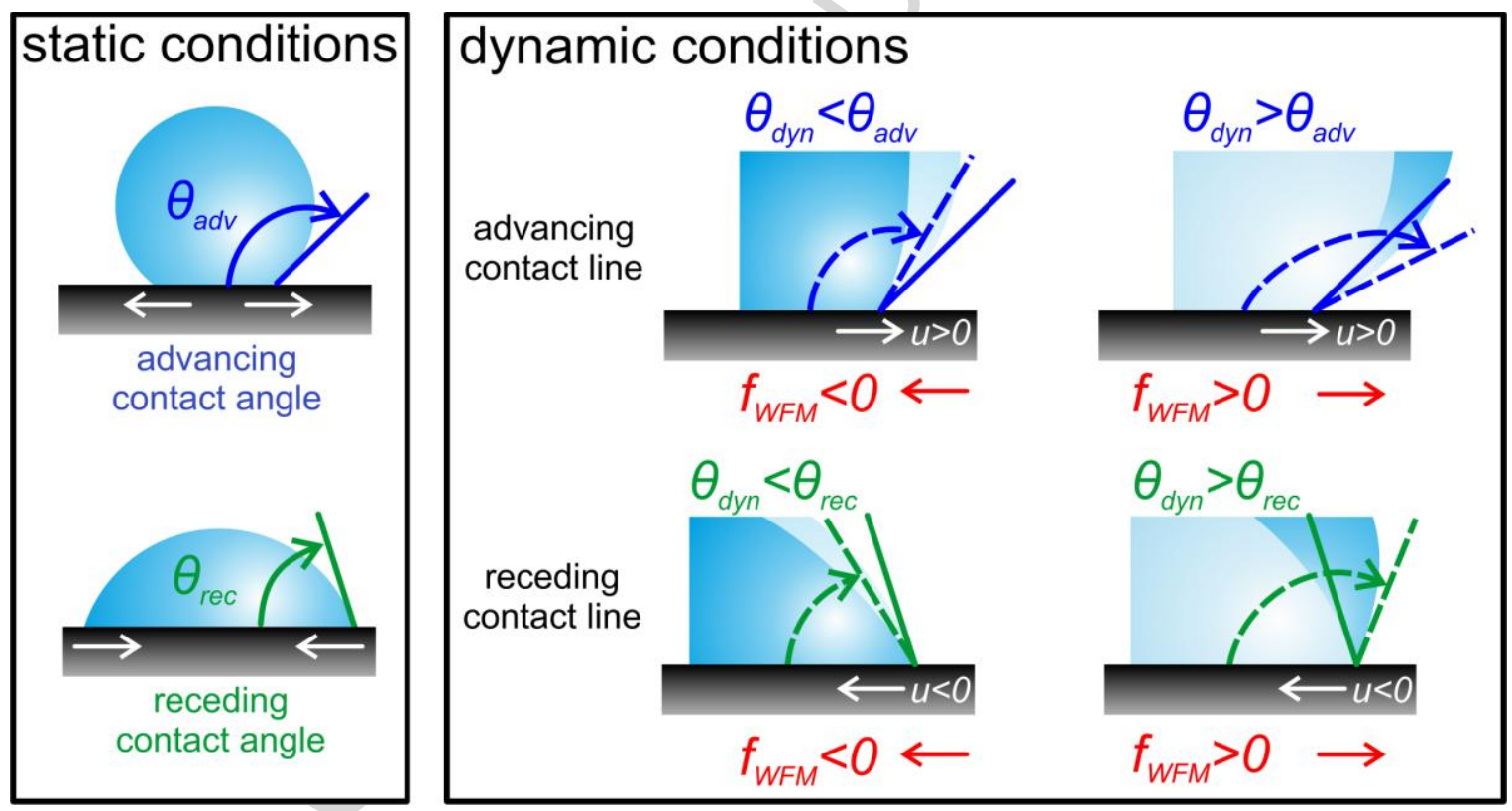

Figure 2. Direction of the proposed adhesion term at the contact line for dynamic conditions: $f_{W F M}$ points to the left/right until $\theta_{\mathrm{eq}}$ is reached.

More specifically, if one takes a closer look at eq. 11, it is clear that the magnitude of this "adhesion" force is dependent on the dynamic contact angle at the three phase contact line, and more precisely its difference from the equilibrium contact angle. Therefore, for an advancing contact line, if the dynamic contact angle is lower than the equilibrium one, then this force shall have a direction opposite to droplet further spreading so that that the rim can reach its equilibrium angle (Figure 2). On the other hand, if the dynamic contact angle is larger than the equilibrium one, the force would have the opposite direction, tending to accelerate the rim. For the receding phase, the concept is the same, as can be seen in Figure 2. In physical terms, the application of this extra "adhesion" force, only at the contact line, tends to restore the droplet shape to its static condition, i.e. to form an equilibrium angle, by either promoting or counterbalancing droplet further spreading/recoiling. Furthermore, it is clear that as the difference between the dynamic contact angle and the equilibrium gets higher, the force magnitude is 
higher and its effect on droplet motion is stronger. Finally, since an axisymmetric case is considered, the force is proportional to the droplet radius (as one can verify from eq. 11).

The basic differentiation of the proposed model relative to previous works is the incorporation of this force into the Navier-Stokes momentum equation instead of imposing a specific equilibrium or dynamic contact angle value as a boundary condition; from now on this approach will be referred as Wetting Force Model (WFM). In that way the microscopic change of the dynamic contact angle, as well as the macroscopic temporal evolution of the phenomenon, will change according to how this force aids or counteracts the droplet spreading onto the solid substrate. This stress term, therefore, would dynamically affect the formation of the dynamic contact angle in relation to its interaction with the standard surface tension force and viscous dissipation near the wall.

An additional advantage of this approach, when compared to the standard boundary condition (BC) approach, based on the work of Brackbill [69], is that by this methodology, one can really measure the temporal evolution of the dynamic angle the droplet forms, and compare this with available experimental data. This is shedding light on the complicated, not yet fully understood, underlying physical mechanisms controlling droplet impingement on any type of either flat or non-flat solid surfaces.

At this point, it should be mentioned that in their work, Sikalo et al. [50] in 2005 had already presented a study in which they impose as boundary condition the wetting force instead of a contact angle. In their simulations, however, this was only an alternative way to apply the Hoffmann's law: the force applied at the contact line was calculated as function of the dynamic contact angle, which was derived from using the Hoffman's law, or alternatively by the theoretical solutions based on creeping flow in the neighborhood of the contact line. In this study, the force does not depend on any law or empirical correlation, but relies on the value of the instantaneous contact angle.

\section{Mathematical modeling}

\subsection{Volume of Fluid Method}

For interface tracking, the Volume of Fluid method (VOF), which is appropriate for the simulation of fluid flow between two immiscible fluids, is used. VOF was first proposed by Hirt and Nichols [77] and considers for the solution of the full Navier-Stokes equations for mass and momentum conservation in conjunction with the advection of a scalar quantity, named as colour function or volume fraction $(\alpha)$. The Volume Fraction, $\alpha$, is defined as the percentage of volume covered by the liquid phase within a computational cell in respect to the total volume of the cell. In mathematical terms, $\alpha$ takes the value of zero if the computational cell is covered only by gas, and the value of one if the cell is completely covered by liquid. For in-between values, the cell lies in a region called the "interface" between the two fluids. The definition of this region does not apply to a real-life existing quantity, but in numerical terms exists, due to the precondition of a continuous solution of the governing numerical equations. These equations namely the mass, momentum conservation equations and volume fraction advection, which are the standard ones for V.O.F. simulations as can be as well found in $[66,78]$, with the addition of the

WFM term, $\vec{f}_{\text {WFM }}$, are presented in this study: 


$$
\begin{aligned}
& \frac{\partial \rho \vec{u}}{\partial t}+\nabla(\rho \vec{u} \otimes \vec{u}-\vec{T})=\rho \vec{g}+\vec{f}_{\sigma}+\vec{f}_{W F M}^{v} \\
& \frac{\partial \alpha}{\partial t}+\nabla(\vec{u} \alpha)=0
\end{aligned}
$$

The solution of the aforementioned equations, in axisymmetric form, was performed in the commercial package of ANSYS FLUENT. The difference of VOF in comparison to other techniques is that a single momentum equation is solved for the two phases (gas - liquid), where the fluid properties are updated according to the volume fraction value of the cell:

$$
\mu=\alpha \mu_{l i q}+(1-\alpha) \mu_{g a s}
$$

$\rho=\alpha \rho_{\text {liq }}+(1-\alpha) \rho_{\text {gas }}$

Surface tension term $\vec{f}_{\sigma}$ in the momentum equation is taken from the work of Brackbill et al. [69]:

$$
\vec{f}_{\sigma}=\sigma \frac{\rho \kappa \nabla \alpha}{\frac{1}{2}\left(\rho_{\text {liq }}+\rho_{\text {gas }}\right)}
$$

where " $\mathrm{K}$ " is the curvature of the free surface and is approximated as the divergence of unit surface normal, i.e.

$\kappa=\nabla \cdot \hat{n}$

$\hat{n}=\frac{\nabla \alpha}{|\nabla \alpha|}$

For a more accurate calculation of curvature, the unit surface normal vectors at the computational nodes are used. Pressure-velocity coupling is achieved by adopting a coupled approach [79] for which momentum and pressure-based continuity equations are solved together, while the Rhie-Chow pressure dissipation terms are included in the face mass fluxes [79]. Discretization of the momentum flux term is achieved by a second-order upwind scheme, while for the time discretization an first-order implicit approach is followed (explicit for volume fraction advection, eq. 13). The time-step of the calculations is varying for all simulations, so that the Courant number can be kept at around 0.25 .

The flux term of volume fraction equation (eq. 13) needs special care, so that diffusion can be avoided and physical results can be obtained. In literature, there are a lot of different ways of achieving a sharp interface, namely [80-82]. However, any special treatment falls out of the aim of the present work, and should be addressed in future works (e.g. decrease of spurious velocities [83]). In this study, the CICSAM [84] discretization scheme was used, which is highly compressive. CICSAM has been proved to provide a reliable way of predicting two-fluid phenomena concerning droplet impingement on a wall film, droplet 
collisions $[78,85,86]$ and impingement onto heated surfaces as reported in the works of researchers from the author's group $[52,53,65-67]$.

\subsection{Implementation of Dynamic contact angle models and Wetting Force Model (WFM)}

\subsubsection{Dynamic contact angle models}

The dynamic contact angles, associated with the quasi-dynamic Adv-Rec, Kistler and Shikhmurzaev models, are implemented in the VOF model using a similar approach to the one presented in Brackbill et al. (eq. 53) [69] and Ubbink (eq. 2.22) [84]. The main idea lies on the use of a prescribed value of the contact angle $\theta$ at the wall as a boundary condition. Then based on this value, the normal to the interface unit vector at the wall boundary cells $\hat{n}$ is rotated, according to the following equation:

$\hat{n}=\hat{n}_{w} \cos \theta+\hat{n}_{t} \sin \theta$

so that the interface can form the prescribed angle $\theta$, when in contact with the wall. Figure 3 is presented for better understanding of eq.19 concept and notation.

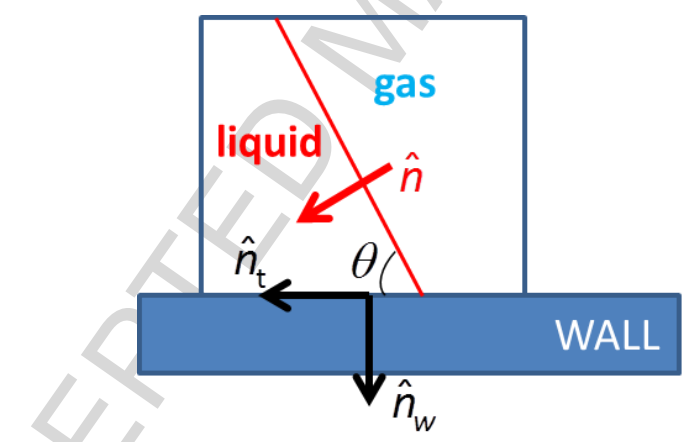

Figure 3. Unit vectors on the wall face, as well as unit free surface normal vector.

In particular, the rotation of this vector influences the interface curvature calculation (eq. 17) at the spreading droplet rim boundary cell, and in turn the surface tension force (eq. 16) applied at the cells.

The calculation of the contact line velocity is based on the actual velocity calculated at each computational cell, in the close region of the rim (triple-phase contact region), from which the velocity component parallel to the wall ( $\left.\vec{u}_{\text {contline }}\right)$ is derived, according to eq. 20 :

$\vec{u}_{\text {contline }}=\left(\vec{u}_{\text {cell }} \cdot \hat{n}_{t}\right) \frac{\hat{n}_{t}}{\left|\hat{n}_{t}\right|}$

Although more accurate methods for contact line velocity have been presented in the literature, based on the time derivative of the radius of the wetted area, as in Sikalo et al. [50] and Roisman et al. [41] $\left(\mathrm{u}_{\text {contline }}=\mathrm{dx} \mathrm{x}_{\text {contline }} / \mathrm{dt}\right.$ ), the simple approximation used here can be easily extended to a three-dimensional case. After the contact line velocity is found, the dot product of the velocity vector with the unit free surface normal provides the direction of contact line movement, i.e. if it the rim is advancing or recoiling. contact line direction $=\vec{u}_{\text {contline }} \cdot \hat{n}$ 
If the resulting value is negative, this means that the rim is advancing ( $\hat{n}$ always points inside the liquid phase), while if it is positive, the rim is recoiling.

In ANSYS FLUENT, a User Defined Function for the dynamic angle value is embedded. The function works as follows: a) it loops over all wall boundary cells, b) it calculates at each cell the contact line velocity (eq.20) along with the contact line direction (eq.21) and c) only at the cells where the volume fraction gradient is non-zero, a prescribed contact angle value is provided. In the background, this value changes the value of $\hat{n}$ at the triple -phase contact region (max 3 cells), according to equation 19.

The choice of the non-zero gradient condition is chosen in accordance with Roisman et al. [41], who applied the contact angle boundary condition imposed on cells in the immediate vicinity of the transition region, characterized by the gradient of the VOF function. This is because the volume tracking algorithm provides a transition region, rather than the exact position of the contact line.

\subsubsection{Wetting Force Model (WFM)}

The proposed force term (eq. 11) is implemented into the Navier-Stokes momentum equation (eq. 12) as an extra source term, active only at the boundary cells in contact with the wall, in the vicinity of the interface. The theoretical correct way to implement this contact line force is to apply that only at one point, i.e. at the triple phase contact line. However, when the contact line is represented by more than one cell, especially in the case of droplet impacts on hydrophilic surfaces (low contact angle values), this stress term should also be applied in more than one cell, owed to the diffusion of the interface.

The "default" approach is to add this term at the cells in contact with the wall, where the volume fraction gradient is non-zero (as referred in Section 3.2.1). However, when this criterion was used for the application of the WFM, an unphysical velocity field was predicted at the contact line region, especially at the time instants when the droplet stopped advancing, right before the initiation of the recoiling phase.

To be more precise, at the time instant when the droplet stops advancing, the stress term which is applied at the contact line cells is such that the rim turns according to the receding contact angle. This results in the introduction of a force pointing towards the interface outward direction in numerous cells (max 3 ), which affects the local flow field and culminates in the unphysical breakup of small secondary droplets, Figure 4.

$\mathrm{t}=9.2 \mathrm{~ms}$

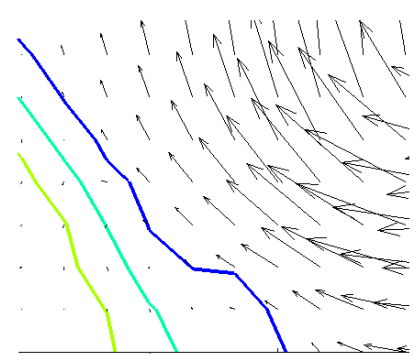

$\mathrm{t}=9.3 \mathrm{~ms}$

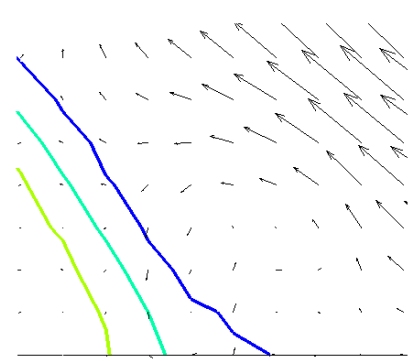

$t=9.4 \mathrm{~ms}$

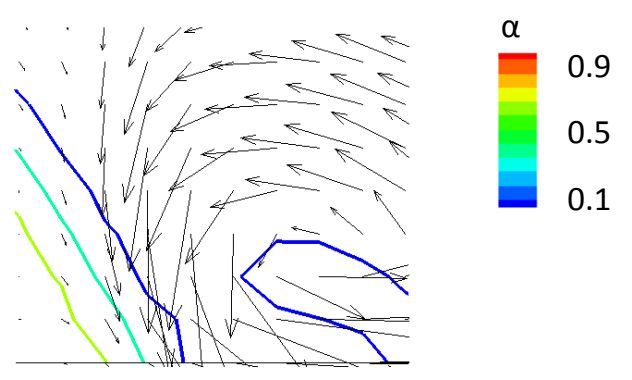

Figure 4. Unphysical diffusion of volume fraction field, followed by the breakup of small secondary droplet observed when the Wetting Force is applied in a wide region around the contact line. Velocity vectors are plotted in the same manner for all pictures. The numerical tests have been performed for the conditions of Case 9 . 
As the contact angle hysteresis gets higher, this unphysical phenomenon becomes more intense. In order to avoid such unphysical behaviour, two alternative strategies can be implemented:

- calculating the contact line velocity as the mass weighted average of all neighbouring cells to the reference cell, so that the contact line velocity can be closer to the liquid phase velocity;

- considering, as an additional criterion for the application loci of the adhesion force, all the interface cells, i.e. the cells with the volume fraction ranging lower than a given small value for the gas phase and higher than a threshold value for the liquid phase.

The first test proved to be unsuccessful, while the second appeared valid for all cases examined (a large range of parameters), using the threshold values of 0.05 and 0.95 as the most representative for interface tracking.

The magnitude of $f_{W F M}$ which is implemented in the momentum equation is given by the following expression:

$\vec{f}_{W F M}^{v}=d \vec{f}_{W F M} / V_{c e l l}=\sigma\left[\cos \left(\theta_{e q}\right)-\cos \left(\theta_{d y n}\right)\right] r / V_{c e l l}$

where

$\theta_{e q}= \begin{cases}\theta_{\text {adv }} & \text { if } \mathrm{u}_{\text {conline }} \geq 0 \\ \theta_{\text {rec }} & \text { if } \mathrm{u}_{\text {conline }}<0\end{cases}$

This stress term is divided by the cell volume in order to be consistent with the momentum equation (eq. 12). The equilibrium contact angle during the spreading phase is assumed to be equal to the advancing contact angle, $\theta_{a d v}$, whereas during the recoil phase the equilibrium contact angle is equivalent to the receding contact angle, $\theta_{\text {rec }}$. $\theta_{a d v}$ and $\theta_{\text {rec }}$ are measured experimentally using the standard sessile drop method, i.e. while expanding and retracting a drop quasi-statically on a horizontal surface; their difference, $\Delta \theta=\theta_{\text {adv }}-\theta_{\text {rec }}$, is the contact angle hysteresis. The method, based on which this dynamic contact angle is calculated, is crucial as it determines the magnitude of this force, and therefore its contribution to the net force. In this work, the dynamic contact angle was calculated from the slope of the normal to the free surface $(\nabla \alpha)$ in the region near the contact line, as shown in Figure $5 \mathrm{c}$.

At this point, the numerical procedure followed for the implementation of the wetting force is described:

i. The contact line velocity (eq.20) and contact line direction (eq. 21) are found for all cells in contact with the wall, where the volume fraction ranges in-between 0.05 and 0.95 . For these cells, it is identified whether the contact line is advancing or recoiling.

ii. Subsequently, advancing or receding contact angles are inserted in eq. 22, in the place of $\theta_{\text {eq }}$.

iii. The resulting source term is inserted in the momentum equation (eq. 12). For the cases examined in this paper, this additional stress term is inserted only in the radial velocity equation, since an axisymmetric approach is followed.

The particular case of a non-axisymmetric drop sticking to the substrate (with $u_{\text {conline }}=0$ and a nonuniform distribution of contact angles on the entire contact line) should be treated in a different, 
specific way and it is not considered in the present paper. More specifically, in case of a sticking drop, e.g. a drop either under shear flow or on a tilted surface, the drop has a non-axisymmetric shape with a non-uniform distribution of contact angles. This causes the generation of a net adhesion force, which opposes to the external force (e.g. aerodynamic forces or gravity) and prevents drop motion. Predicting the onset of drop motion, when external forces overcome drop capillary adhesion, is not trivial, and can be complicated by the presence of surface local heterogeneities, as recently addressed by Varagnolo et al. [87] for the case of chemical heterogeneous surfaces with numerical simulations based on the lattice Boltzmann models. In the present paper, the main objective was not to evaluate the static adhesion force term, but to propose a model for the capillary adhesion force acting at the contact line in nonstationary and non-equilibrium impact conditions. To further extend the present work to study static and close-to-equilibrium conditions, such as the case of drops impacting on high hysteresis or tilted surfaces, one possibility could be to implement an adhesion force for the static case, such as:

$\vec{f}_{W F M}^{v}=\sigma \cos \left(\theta_{\text {eq }}\right) r / V_{\text {cell }}$, if $\left|\mathrm{u}_{\text {conline }}\right|<\varepsilon$

where $\varepsilon$ should be chosen near to the wetting velocity and generally $r$ is the local curvature radius on the surface plane. This "static adhesion force" plays a role when the drop spreading stops and may have an influence both for the stick and slip behavior or for the time at the maximum spreading (Antonini et al. [38]). It is important to note that this force could also capture specific three-dimensional phenomena like the formation of lobes and the wavy rim on the surface.

\subsection{Computational Domain, Boundary conditions, grid refinement}

In Figure 5a, the basic 2-D axisymmetric domain, comprising of $60 \times 60$ identical quadrilateral cells is shown, where local grid refinement of the region around the droplet has been applied. This adaptive local grid refinement technique is selected in order to increase the numerical accuracy as much as possible, with the minimum computational cost, as will be discussed further in the following section. The initial wall-droplet distance, shown in Figure $5 b$, is selected to be one droplet diameter $\left(D_{0}\right)$ long so the gas fluid flow around the droplet can develop in a physical way, just before droplet impact onto the surface. The initial droplet velocity and diameter are defined as $U_{0}$ and $D_{0}$ respectively. Gravitational acceleration is directed towards the positive values of $x$ axis. It is very important to note that the initial domain for all computations, conducted in this paper, was the same, where the spatial coordinates ( $x$ axial coordinate, $y$-radial coordinate) ranged between: $X, Y=0 \div 0.015 \mathrm{~m}$. Therefore, for the wide range of simulated cases presented in Table 2 , the total length of the domain boundaries range between $\left(4 \div 6.6 D_{0}\right)$.

In Figure 5b, a zoomed area of the applied numerical grid around the moving droplet, along with the high resolution of the grid are shown. This figure serves for the better understanding of the dynamic grid refinement technique. According to this technique, which is based on the work of Theodorakakos et al. [88], the mesh is dynamically refined every twenty time-steps in a prescribed distance from the interface. Numerically, this is achieved by a) looping over all cells of the domain once, b) finding the isoline of $\alpha=0.5$ and then c) looping over the cells as many times as the isoline cells, in order to mark the ones that lie within a specified distance from them. In this study, this distance was selected to be $D_{0} / 5$, 
which was found to be far enough from the interface, so that the gradients of volume fraction are computed in the region with the denser grid cells. In that way, it is guaranteed that the computed surface tension term that will be inserted in the momentum equation will always have the highest level of accuracy.

Since such grid treatment is not given as a standard user option in ANSYS/FLUENT software, implementation of this technique was achieved through a User Defined Function (UDF). The method of refinement was based on the hanging node adaption, available in ANSYS FLUENT. In this work, four levels of refinement were used, achieving a minimum cell size of around $\Delta x_{\text {initial }} / 2^{4}=15.6 \mu \mathrm{m}$. This translates to approximately $72-120$ cells along droplet radius for all the cases examined, which is in accordance to the current state-of-the art maximum grid resolutions applied in such cases. The application of this technique allows important computational time to be saved, as a uniform grid would require to be comprising of 921,000 cells uniformly distributed over the whole computational domain; for comparison, here only 24,672 cells are required for a typical droplet with initial diameter $D_{0}=2.75 \mathrm{~mm}$, for the same grid resolution around the interface to be achieved.

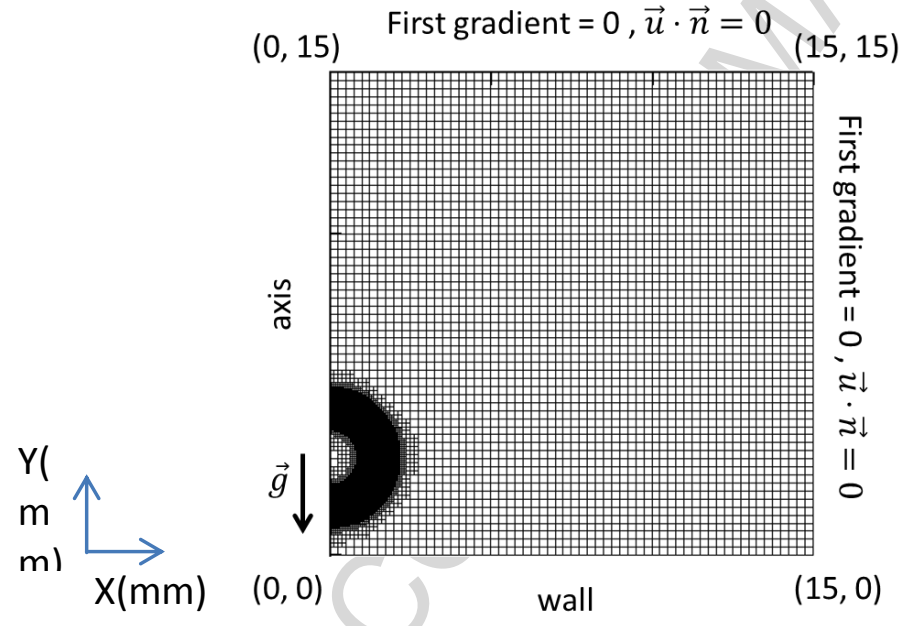

a

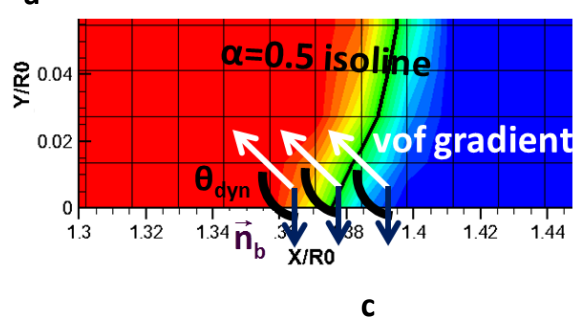

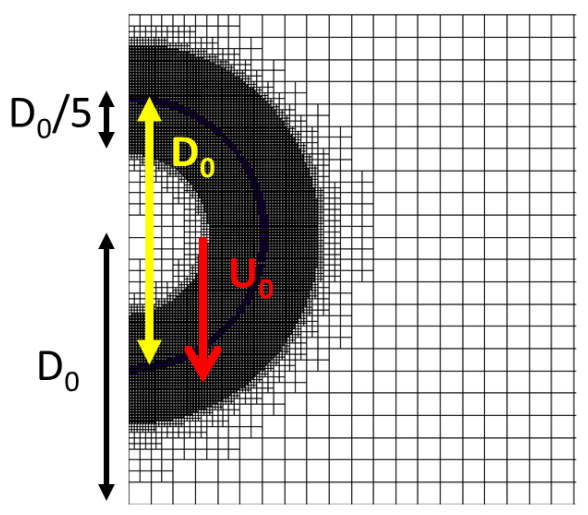

wall

b of $\theta_{\text {dyn }}$ for the WFM.

\subsection{Simulated cases}

Nine cases are used for the validation of the proposed model; the corresponding conditions are summarized in Table 2 and concern the impingement of a water droplet onto both hydrophilic and hydrophobic surfaces. A wide range of We numbers ranging from as low as 0.2 up to 120 have been examined. Cases 1-4 refer to the impingement on hydrophobic surfaces, while Cases 5-9 concern 
hydrophilic surfaces. Case $6(\mathrm{We}=0.2)$ has been included in order to compare its results against those of Case 5, which also refers to a very low Weber number; it is known that for such very low Weber numbers, peculiar post-impingement liquid shapes are forming, as it will be further presented in a following section. The properties for liquid water and air that were used in the simulations are $\rho_{\text {liq }}=998.2$ $\mathrm{kg} / \mathrm{m}^{3}, \mu_{\mathrm{liq}}=1.003 \cdot 10^{-3} \mathrm{~kg} / \mathrm{ms}, \rho_{\mathrm{gas}}=1.225 \mathrm{~kg} / \mathrm{m}^{3}, \mu_{\mathrm{gas}}=1.7894 \cdot 10^{-5} \mathrm{~kg} / \mathrm{ms}$.

\begin{tabular}{|c|c|c|c|c|c|c|c|c|c|c|c|c|}
\hline $\begin{array}{l}\text { Cases } \\
\text { Run }\end{array}$ & $\begin{array}{c}U_{o} \\
(\mathrm{~m} / \mathrm{s})\end{array}$ & $\begin{array}{c}D_{o} \\
(\mathrm{~mm})\end{array}$ & $\begin{array}{c}\theta_{\mathrm{adv}} \\
\left(^{\circ}\right)\end{array}$ & $\begin{array}{c}\theta_{\text {rec }} \\
\left({ }^{\circ}\right)\end{array}$ & We & $\operatorname{Re}$ & Exp & Sim & $\begin{array}{c}\text { Pasand } \\
\text { Theory } \\
\text { [18] }\end{array}$ & $\begin{array}{c}\text { WFM- } \\
\text { Exp }\end{array}$ & $\begin{array}{l}\text { ev } \\
\text { Pas- } \\
\text { Exp } \\
\end{array}$ & Ref \\
\hline $\begin{array}{c}\text { 1. LW-HCA- } \\
\mathrm{HH}\end{array}$ & 0.48 & 2.50 & 120 & 65 & 8 & 1200 & 1.50 & 1.71 & 1.92 & 14.2 & 28.0 & [41] \\
\hline $\begin{array}{c}\text { 2. MW-HCA- } \\
\mathrm{HH}\end{array}$ & 1 & 2.28 & $\begin{array}{c}107 \\
\left({ }^{*} 90\right)\end{array}$ & 77 & 32 & 2280 & 2.29 & 2.42 & 2.59 & 5.7 & 12.9 & [54] \\
\hline $\begin{array}{c}\text { 3. MW-HCA- } \\
\text { LH }\end{array}$ & 1.18 & 2.75 & 105 & 95 & 53 & 3245 & 2.62 & 2.85 & 2.95 & 8.8 & 12.4 & [30] \\
\hline $\begin{array}{c}\text { 4. HW-HCA- } \\
\text { LH }\end{array}$ & 1.64 & 2.45 & 105 & 95 & 91 & 4018 & 3.1 & 3.30 & 3.29 & 6.5 & 6.2 & [50] \\
\hline $\begin{array}{l}\text { 5. VLW- } \\
\text { MCA-LH }\end{array}$ & 0.22 & 2.40 & $70^{*}$ & 70 & 2 & 527 & 2.4 & 1.69 & 2.46 & -29.6 & 2.4 & [40] \\
\hline $\begin{array}{l}\text { 6. VLW-LCA- } \\
\text { LH }\end{array}$ & 0.08 & 2.30 & $31^{*}$ & 31 & 0.2 & 184 & 1.96 & 1.86 & 5.00 & -5.1 & 155.0 & [32] \\
\hline $\begin{array}{c}\text { 7. LW-MCA- } \\
\text { LH }\end{array}$ & 0.76 & 2.40 & $70^{*}$ & & 9 & 1821 & 2.8 & 2.48 & 2.88 & -11.4 & 2.7 & [40] \\
\hline $\begin{array}{c}\text { 8. MW-LCA- } \\
\text { LH }\end{array}$ & 1.18 & 3.04 & 10 & & 50 & 3587 & 3.31 & 3.62 & 4.22 & 9.4 & 27.6 & [30] \\
\hline $\begin{array}{c}\text { 9. HW-MCA- } \\
\mathrm{HH}\end{array}$ & 1.50 & 3.76 & $\begin{array}{c}60 \\
(* 34)\end{array}$ & 22 & 117 & 5640 & 4.05 & 4.18 & 4.09 & 3.2 & 0.9 & [43] \\
\hline
\end{tabular}

Table 2. Test Cases used for the validation of the new model. Initial conditions are presented, together with results from the WFM, experiment and the theoretical correlations proposed by Pasandideh [18] (eq. 1). For the simplification of Cases, the following abbreviations are used: first the Weber number (W), then the advancing contact angle (CA), and finally the contact angle hysteresis $(H)$, while the symbols $V L, L, M, H$ represent the words very low, low, moderate and high. (* equilibrium angles (Cases2,9). When only the equilibrium angle is given, the advancing/receding angles take this value, as in Cases5-7.)

\section{Results and discussion}

In this section the results obtained are presented. Initially, a brief grid dependency study will be presented together with results for validating the global behavior of the model. Then, detailed predictions for the temporal evolution of the relevant phenomena are presented: Initial predictions for the evolution of the dynamic contact angle are given followed by the corresponding ones for hydrophobic and hydrophilic cases.

\subsection{Grid size verification}

For a thorough validation of the model, and due to the fact that in the present simulations the dynamic contact angle is calculated, the effect of grid size on the numerical results should be investigated, so as to prove that the magnitude of the force exerted, in relation to the surface tension force, is not meshdependent. For that reason, Cases 1 (LW-HCA-HH) and 7 (LW-MCA-LH) were run using six levels of local refinement, achieving a minimum cell size of around $\Delta x / 2^{6}=3.9 \mu \mathrm{m}$. The results for these cases were then compared to the ones obtained with the use of 4 levels of local refinement, which finally have been 
the default choice for this study. Figure 6 presents the temporal evolution of the calculated dynamic contact angle, measured from the volume fraction 0.5 iso-line passing from the reference cell (one cell in contact with the wall), after the application of the Wetting Force Model using 4 (default) and 6 levels of local refinement respectively, in comparison to the available experimental values. Moreover, for Case1 (LW-HCA-HH) the experimental data of Roisman et al. [41] are well fitted. It is clear that the effect of grid size is minimal both for hydrophobic (Figure 6a) and hydrophilic (Figure 6b) surfaces, even if in the latter case, the results for the dynamic contact angle are slightly improved by the application of 6 levels of local refinement. The "noise" observed in Figure 6a can be attributed to the very small cell size, in relation to the sign change of the applied wetting force. More precisely, as the reference cell size (where contact angle is measured) gets smaller, the interface is more prone to direction changes owed to a) velocity field fluctuations and $b$ ) the application of an updated stress magnitude at each time instant. Additionally, one should not forget that at each time instant the curvature of the interface changes, which in turn affects the induced velocity field and therefore the formed contact angle. This in turn affects the exerted on that cell stress magnitude. Moreover, one could imagine that this oscillating behavior, presented in Fig.4a, may resemble the pinning of droplet during its spreading between two values as stated by [89], but this should be further investigated.

The same behavior is observed for Case7 (LW-MCA-LH), at the time when the dynamic contact angle reaches the set equilibrium value $\left(\theta=70^{\circ}\right.$, Table 2$)$. On the whole, the numerical results are not considered to be highly dependent on the level of local grid refinement and given the fact that 6 levels of local refinement is computationally too expensive, the numerous cases presented in this paper are run using 4 levels of local refinement.

\section{Case1. LW-HCA-HH}

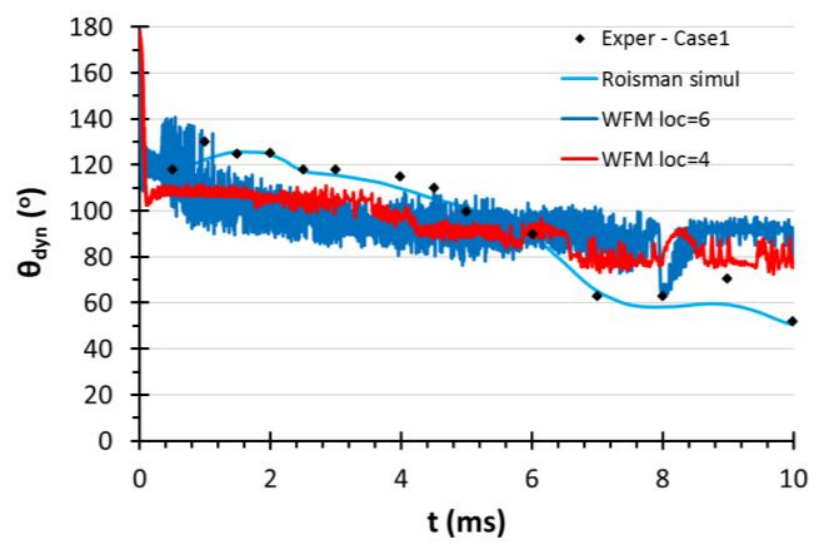

a

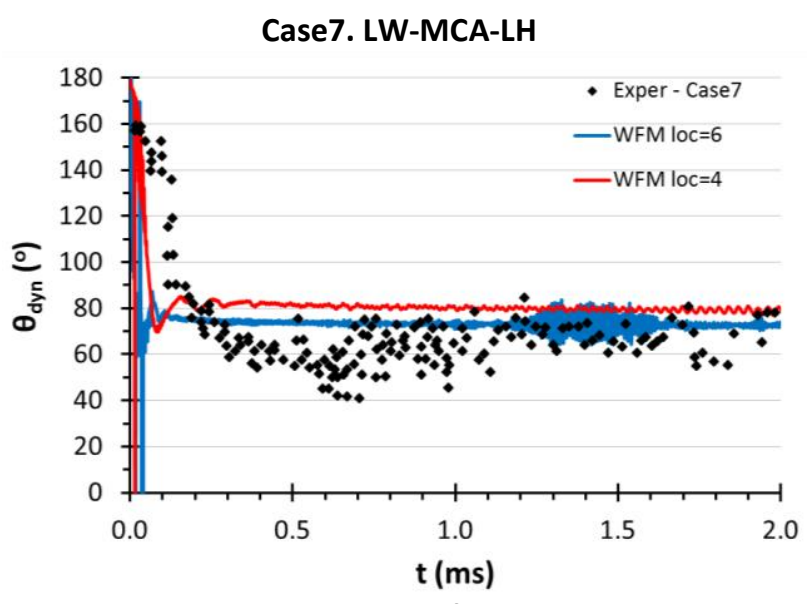

b

Figure 6. Effect of grid size for Cases 1. LW-HCA-HH and 7. LW-MCA-LH of Table 2 a) $\theta_{\text {dyn }}$ as a result of the WFM simulation using 4 and 6 levels of local refinement in comparison with the experimental values and simulation results of Roisman et al. [41]. b) $\theta_{\text {dyn }}$ as a result of the WFM simulation using 4 and 6 levels of local refinement in comparison with the experimental values of Roux et al. [40]. The symbol "rm" in the graphs stands for remeshing.

\subsection{Global performance of the model}

Figure 7a presents the maximum droplet spreading as calculated from the WFM simulation against the corresponding experimental values. It is clear that the new model behaves satisfactorily for all cases, with the exception of Case5 (VLW-MCA-LH). Similarly, Figure 7b presents the maximum dimensionless 
spreading as a function of Weber number; here 3 sets of data have been included: a) WFM predictions, b) experiments and c) the estimates of the Pasandideh's correlation (eq. 1). The results obtained by application of the Pasandideh's equation overestimate the experimental droplet maximum spreading ${ }^{1}$, while the WFM model predicts accurately the impingement onto dry hydrophobic surfaces, even if it is less accurate for the hydrophilic ones, particularly for moderate Weber numbers. Concerning the hydrophobic surfaces, results of the WFM slightly overestimate (approx. 10\%) the experimental findings. On the other hand, for hydrophilic surfaces, not a monotonic overestimation or underestimation of the model predictions against experimental data is tracked.
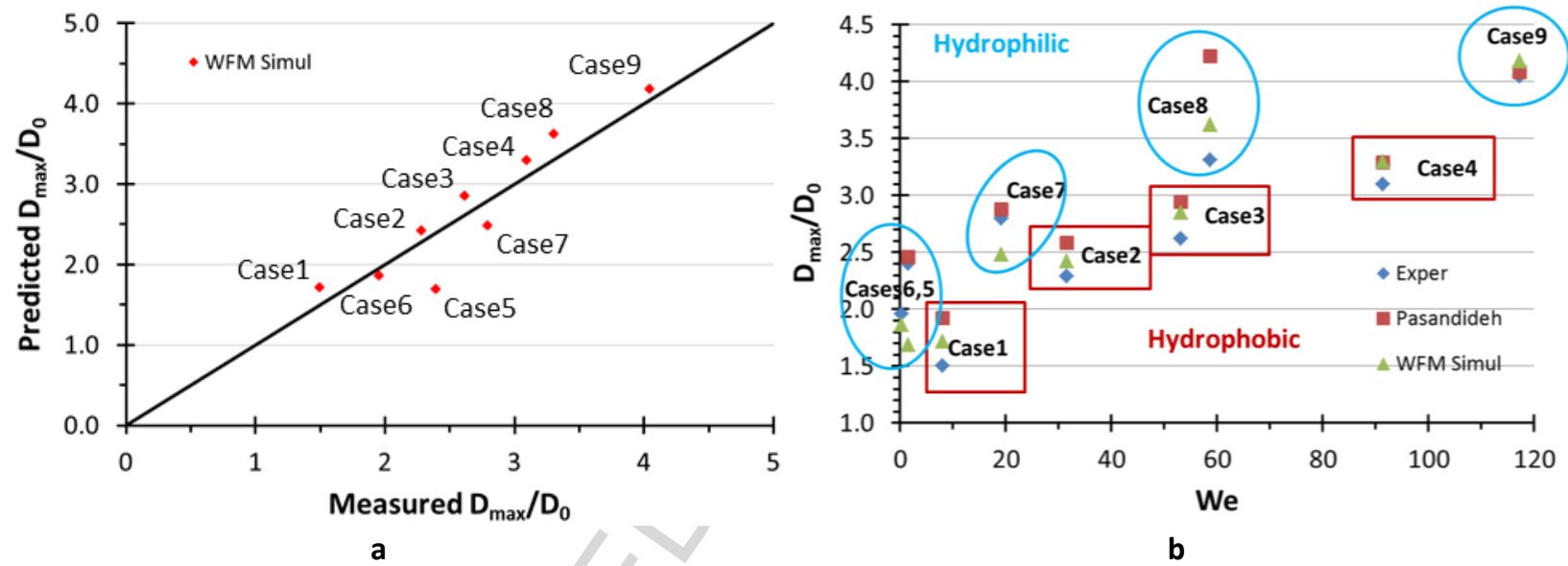

Figure 7. Summary of simulation results with the use of WFM model. a) Droplet maximum spreading plotted against the experimental values, b) Maximum spreading plotted against experimental values and results of eq (1).

Being able to predict the droplet maximum spreading for a variety of contact angles and Weber numbers, it can be claimed that the WFM can be trusted; moreover, a simple grid dependency study showed that the results are not mesh affected. After that, a more detailed comparison of the model predictions will follow in the forthcoming sections.

\subsection{Dynamic contact angle evolution}

The results of the dynamic contact angle predictions for both hydrophobic and hydrophilic surfaces presented in Figure 6 serve as a sign of validity for the new model (WFM), which as noted above can shed light in the temporal evolution of the phenomenon by not prescribing a contact angle value, but on the other hand predicting it. For hydrophobic surfaces, as stated in the Introduction section, only the study of Roisman et al. [41] exhibits dynamic contact angle measurements and thus can be used for validation; in Figure 6a the predictions of the WFM and experimental data are shown for Case1 (LW-HCA$\mathbf{H H}$ ), accompanied by the corresponding numerical results presented in the work of Roisman et al. [41], who used the Kistler's dynamic contact angle model (eq. 6). The dynamic contact angle predicted by the WFM during the advancing phase is lower than the one measured during the experiments, which is a reason for the over-prediction of maximum spreading (Case1 in Figure 7).

\footnotetext{
${ }^{1}$ It should be mentioned that Pasandideh equation is not applicable for very low Weber numbers, because the assumption of a viscous boundary layer influencing significantly energy dissipation is not valid for very low Reynolds numbers [17]; that is why maximum spread for Case6 (VLW-LCA-LH) is highly overestimated and therefore not included in Figure 7b.
} 
During the initial stage of impingement, as soon as the liquid droplet comes in contact with the surface, $\theta_{\text {dyn }}$ is approximately $180^{\circ}$. Subsequently, the contact angle reduces rapidly to approximately $110^{\circ}$, which is lower than the equilibrium angle accounted for in eq. 22 (i.e. the advancing one, $120^{\circ}$ ) and slowly reduces during the rest of the advancing phase. $\theta_{\text {dyn }}$ then drops suddenly to $80^{\circ}$ at approximately $6.5 \mathrm{~ms}$, which can be set as the initiation of the receding phase; this value is higher than the equilibrium value of $65^{\circ}$ set in Eq. 22. This shows that the application of the wetting force has smaller effect than expected. Contact line velocity exhibits similar behavior (presented in Figure 8). At first, a spike is observed when the droplet hits the surface, followed by an almost exponential reduction of its magnitude with time. The time interval between 5 and $6.5 \mathrm{~ms}$ is considered to be the "hysteresis" time, i.e. the time needed for the contact angle to decrease from values $\sim \theta_{\text {adv }}$ to $\sim \theta_{\text {rec. }}$. During this stage, the contact line velocity fluctuates around zero. At later stages, $t>6.5 \mathrm{~ms}$, contact line velocity exhibits negative values, i.e. the droplet is recoiing, with substantially smaller magnitude than during the advancing phase.

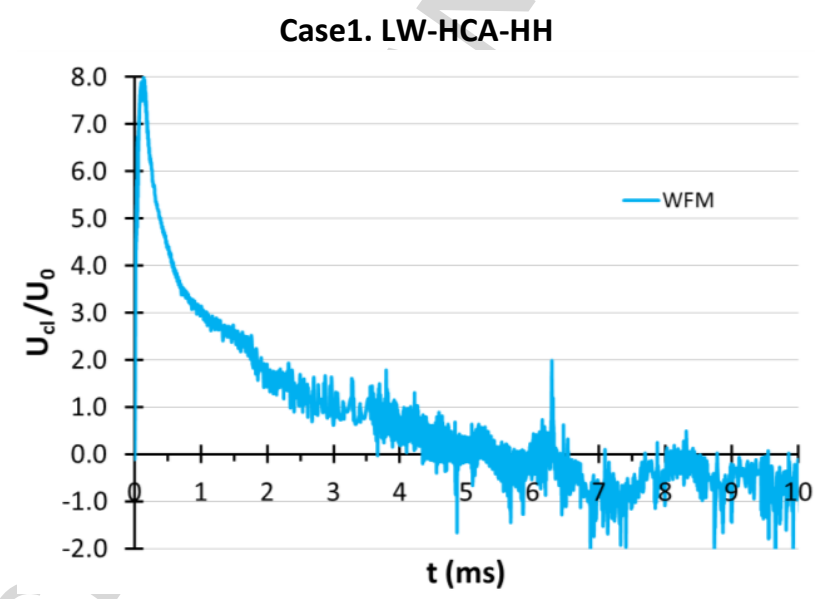

Figure 8. Contact line velocity throughout the impingement for Case1. LW-HCA-HH.

In Figure 6b, the dynamic contact angle for Case7 (LW-MCA-LH) is compared against the experimental values of Roux et al. [40]. During the initial stage of impingement, the contact angle starts with a value again of $180^{\circ}$ and drops again quite fast down to $80^{\circ}$. This trend is also observed in the experimental data; however, the duration of this period is underestimated by the simulation. Afterwards, a constant angle of around $80^{\circ}$ is predicted by the model, while the experimental values lie between $50^{\circ}$ and $80^{\circ}$. This harmony between simulation and experiment is also confirmed by the agreement in droplet shapes depicted in Figure 17, which will be presented in the following sections.

\subsection{Wetting Force Magnitude}

Concerning the magnitude of the WFM, the following Figure reveals its comparison for two reference cases tested (Case 2 and Case 9) against the force exerted by the standard CSF approach, Figure 9.

The wetting force which is applied is of the same order of magnitude with the surface tension force, demonstrating that it can affect in a reasonable manner the interface rim angle of spreading. The main difference between this model and the implementation of the contact angle through boundary condition, as it is observed in Fig. iii is that in the wall boundary cell the induced surface tension force is 
much more intense, which however does not exhibit any significant changes in the macroscopic simulation of the phenomenon of liquid droplet impact on a solid flat surface.

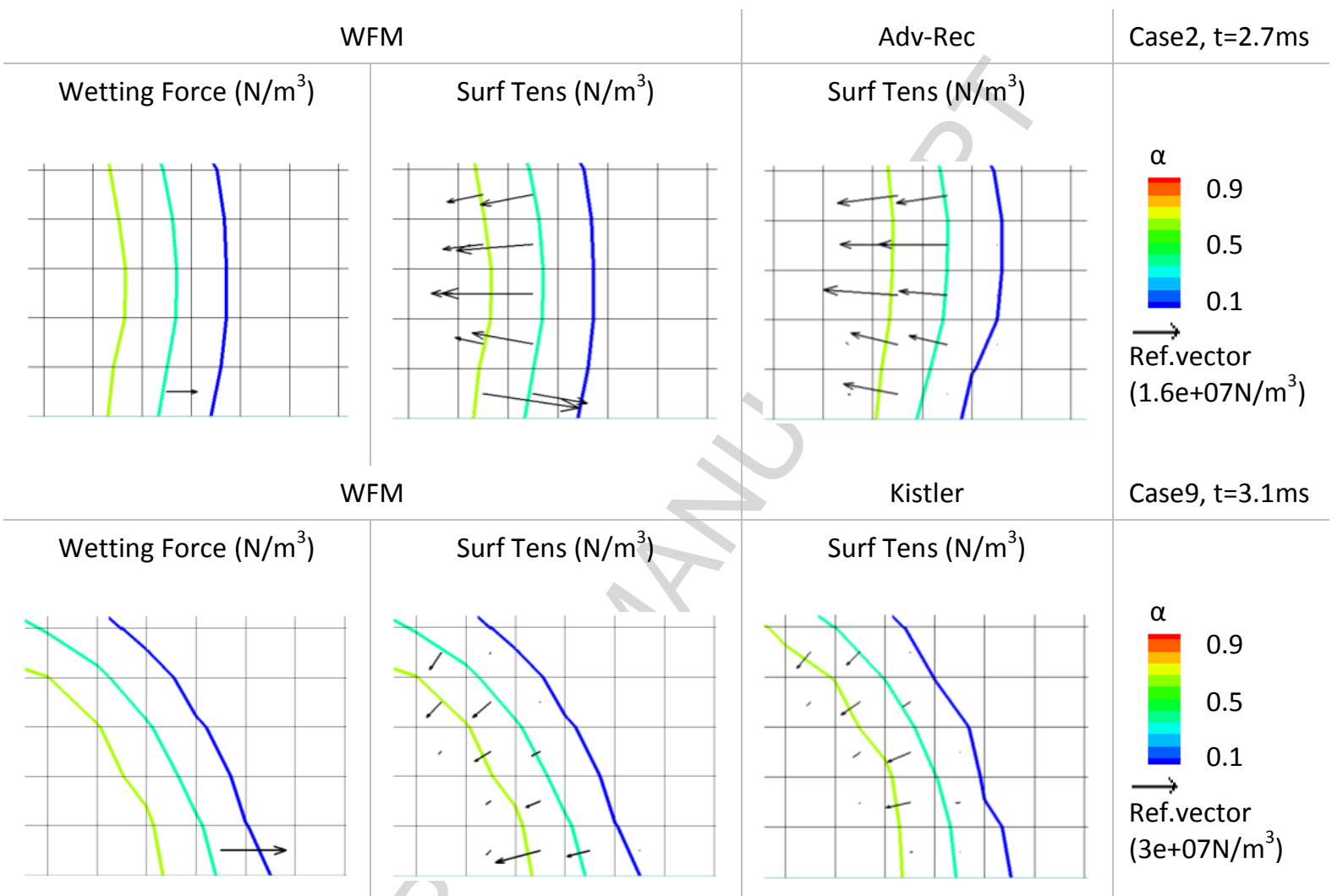

Figure 9 Magnitude of the Wetting Force, as well as surface tension force predicted by the WFM in a random time instant compared to the surface tension force predicted by Kistler's model. Force vectors are plotted in the same manner for both Cases (using the value of $3 e-08 \mathrm{~cm} /$ magnitude).

Additionally, if the WFM was simply turned off, the resulting contact angle at the wall boundary would be approximately $90^{\circ}$, since the applied $\mathrm{BC}$ would have been that of a zero Neumann boundary condition $\left(\nabla \hat{n}_{\mathrm{x}}=0, \nabla \hat{n}_{\mathrm{y}}=0\right)$.

\subsection{Spreading on hydrophobic surfaces}

In Figure 10, the temporal evolution of the impingement of a water droplet onto solid hydrophobic surfaces is depicted for the Cases 1-4 of Table 2. More specifically, results from the application of WFM are compared against the corresponding experimental data; in addition, the predictions obtained using the dynamic contact angle models of Kistler and Shikhmurzaev, as well as those obtained by assuming fixed advancing-receding angles are also indicated. Furthermore, in the same figures, results reported in the literature by other researchers for the same cases and referenced in Table 2 have been also included. Exception to this is Case 3 (MW-HCA-LH), since previous simulation results cannot be found in literature to the best of the author's knowledge; it is noted that the results of Caviezel et al. [55], that also refer to this case, present only comparison against corresponding photographs during the impingement process but the temporal evolution of the spreading radius has not been provided in the corresponding reference. 
Case1. LW-HCA-HH. We $=8$

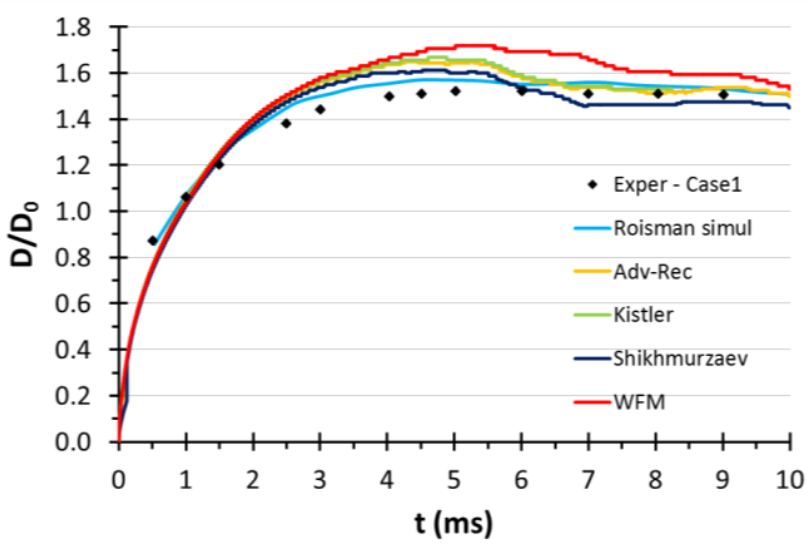

Case3. MW-HCA-LH. $\underline{\text { We }=53}$

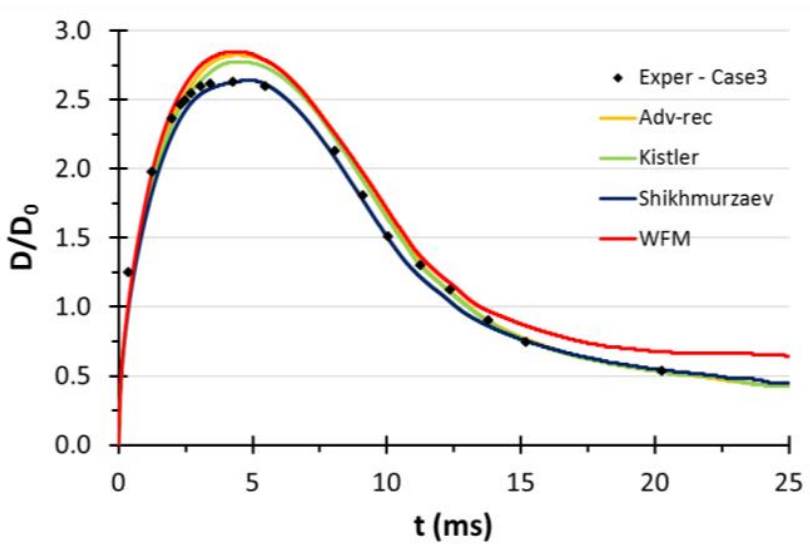

Case2. MW-HCA-HH. We $=32$

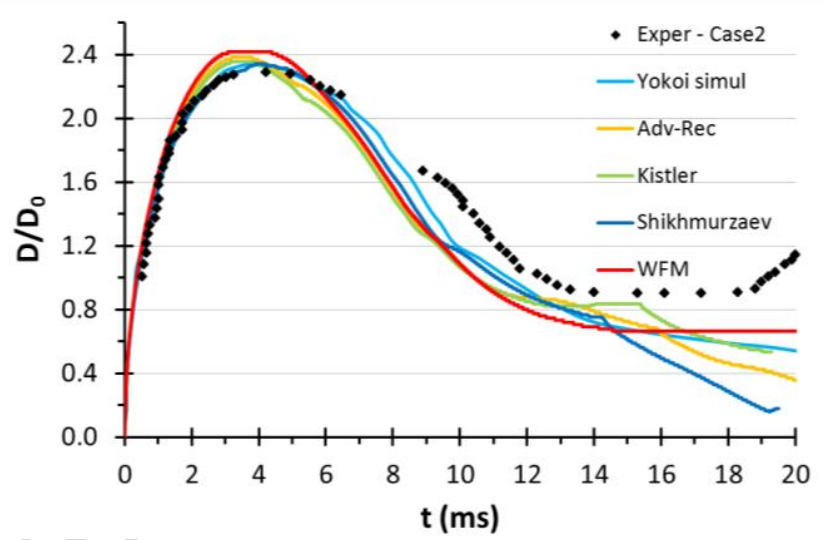

Case4. HW-HCA-LH. We $=91$

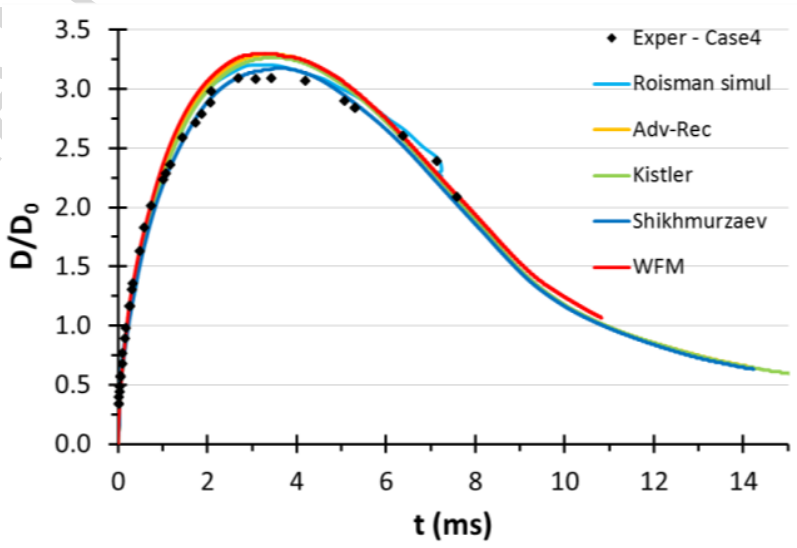

Figure 10. Comparison of WFM results against experiments and respective simulations for the hydrophobic surfaces of Cases1-4 listed in Table 2(where references can be found); corresponding predictions from other dynamic contact angle models found in literature are also indicated.

In general, the Wetting Force Model overestimates slightly the maximum droplet spreading. Given that the proposed model calculates the contact angle rather than using it as a boundary condition, a good agreement between the results derived from the WFM and the experiments can be claimed. It should be noted that contact angle predictions, as provided by the Shikhmurzaev's and Kistler's models as function of the contact line velocity assuming an arbitrary $\theta_{\text {eq }}=90^{\circ}$ can give up to $20 \%$ difference. This suggests that significant differences when predicting the contact angle can be expected with all the previous models.

In Figure 11 and Figure 12 the droplet shape at characteristic times during the impingement process is presented and compared to photographs taken from experiments for the conditions of Cases 3 (MW-HCALH) and 4 (HW-HCA-LH). It is clear that apart from the quantitative validation afore-presented, WFM captures also qualitatively the phenomenon of a water droplet impinging on hydrophobic surfaces. In Figure 11 the spreading and recoiling of the droplet, as well as the break-up of a large satellite droplet for Case 3 (MW-HCA-LH) is predicted. It should be pointed out that at the later stages of the recoiling phase, the simulation shows the same qualitative behavior, but with a trend slightly faster than what the experiment suggests (for example at $t=20.54 \mathrm{~ms}$, in simulation results, the break-up of the satellite 
droplet has occurred, while in the experiment this phenomenon occurs later). Similar results are presented by Caviezel et al. [55], indicating a very good agreement between the results of the two codes. In Figure 8 , the isosurface $\alpha=0.5$ is depicted, where the interior of the droplet is cut in order to present both the dynamic grid refinement technique and velocity vectors throughout the impingement process. Moreover, the pressure of the liquid phase is shown for the boundary wall cells. For the nondimensionalisation of these parameters, velocity magnitude is divided by the initial impact velocity, while pressure is divided by the initial kinetic energy of the droplet, thus defining the pressure coefficient as:

$C_{p}=\frac{P-P_{\infty}}{\frac{1}{2} \rho_{\infty} U_{\infty}{ }^{2}}=\frac{P_{\text {momentum }}}{\frac{1}{2} \rho_{\text {liq }} U_{0}{ }^{2}}$

In this equation, $\mathrm{P}_{\mathrm{oo}}$ and $\mathrm{U}_{\mathrm{oo}}$ are the velocity and pressure on the far field. The pressure difference is given by the value of pressure evaluated in the momentum equation.

TIME WFM

(ms)

$\mathrm{t}=0$

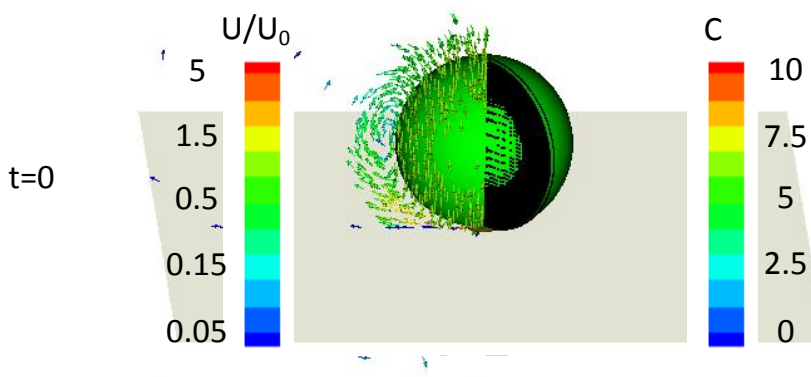

$t=1.31$

$t=3.14$

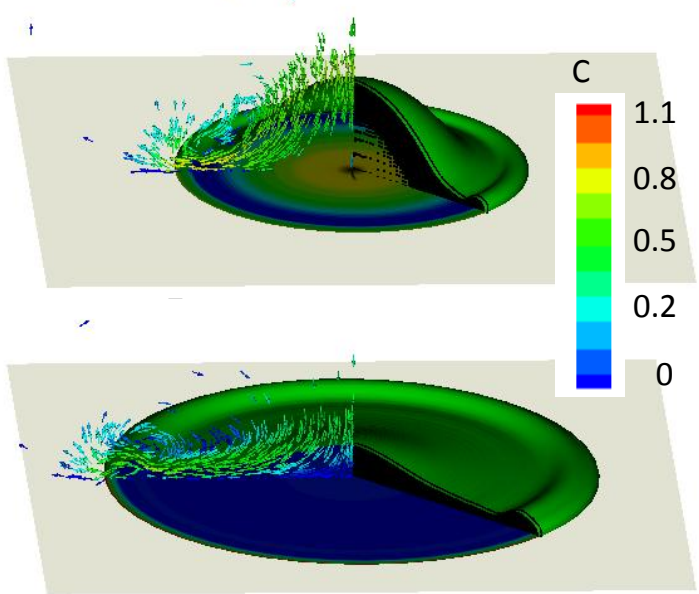

$t=6.02$
Exper (Rioboo et al.) [30]
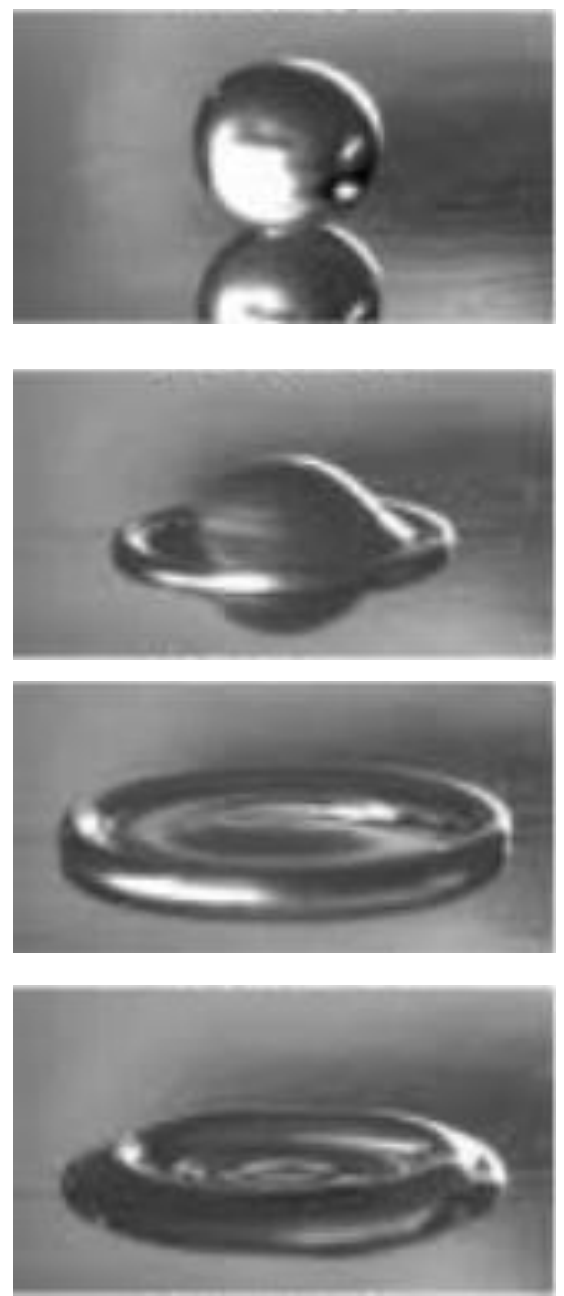

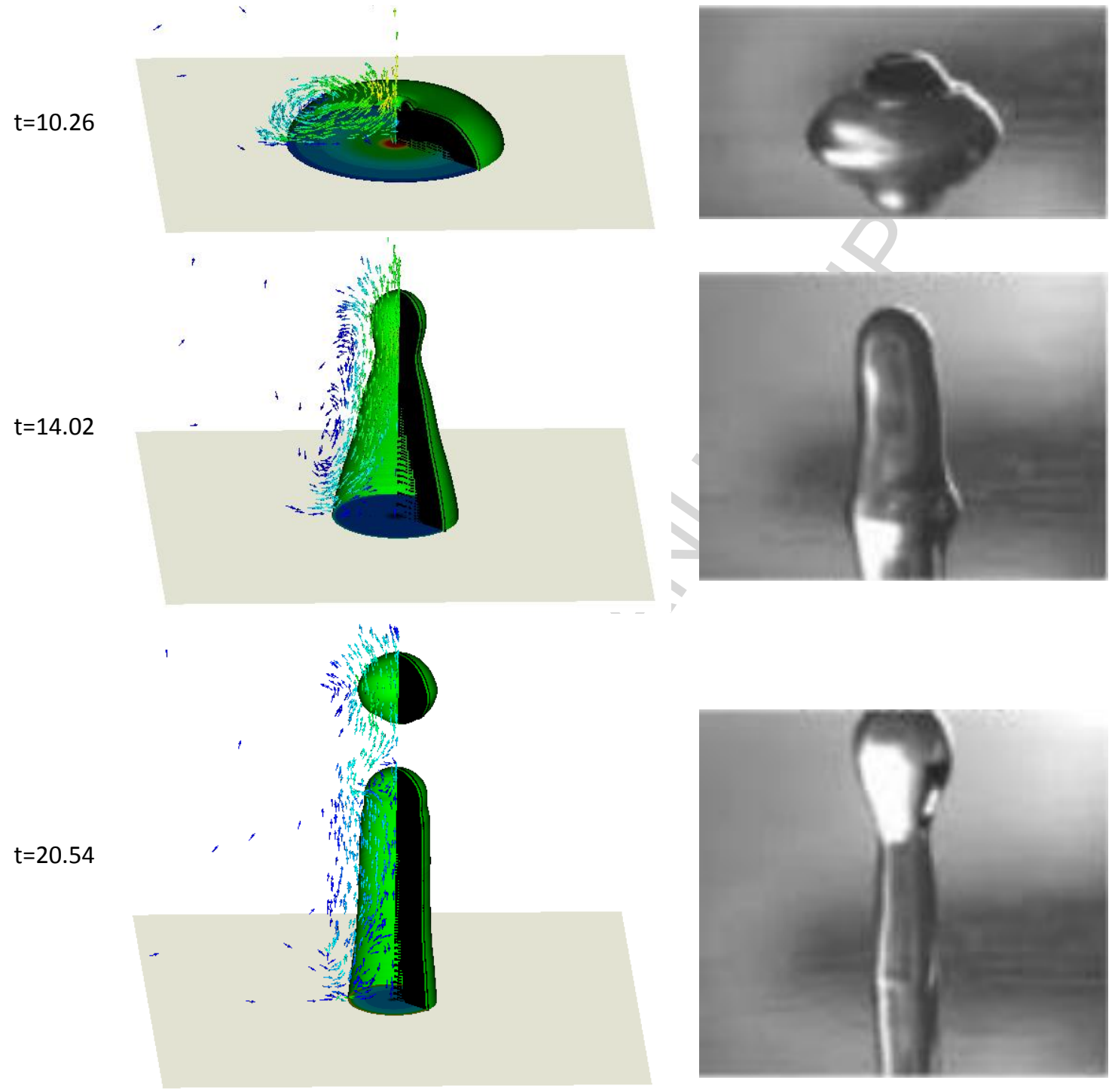

Figure 11. Comparison with experiment for Case 3. MW-HCA-LH (Rioboo et al. [30]). Pictures taken from reference. Results are represented in 3 dimensions, after the revolving of symmetry axis. $C_{p}$ contour and velocity vectors colored by velocity magnitude are plotted. $C_{p}$ values below 0.01 are cutoff. The first $C_{p}$ contour legend applies to the first two pictures, while the second one applies to all the following. Velocity contour is the same for all pictures.

At the initial stage of impingement, $\mathrm{t}=0-1.31 \mathrm{~ms}$, pressure can reach up to 9.7 times of droplet initial kinetic energy, which can be explained by pressure rise due to formation of a dimple upon drop impact [90]. During the rest of the spreading phase and the start of the recoiling phase ( $t=3.14-6.02 \mathrm{~ms}), C_{p}$ is much lower, while its maximum value is observed on droplet rim. As the retraction of liquid mass continues, pressure is building up on droplet center, where the liquid is pressed towards an upward movement. The induced flow field is typical for impingement cases, where during the initial stage of the process, velocity magnitude can reach up to 6 times the initial impact velocity, while during the rest of the advancing and receding phases the maximum velocity magnitude is only 1.5 times the impact velocity. Moreover, the vortex that is formed on the top of droplet rim clearly changes direction between $3.14 \mathrm{~ms}$ and $6.02 \mathrm{~ms}$ marking the initiation of the receding phase. 
In Figure 12, the comparison between pictures taken from the work of Sikalo et al. [50] (Case4 HW-HCA-LH) are compared against the results of the WFM simulation. Again, very good qualitative agreement is observed between simulation and experiment. In this figure, the pressure contours, as well as the induced flow field during the process are presented.
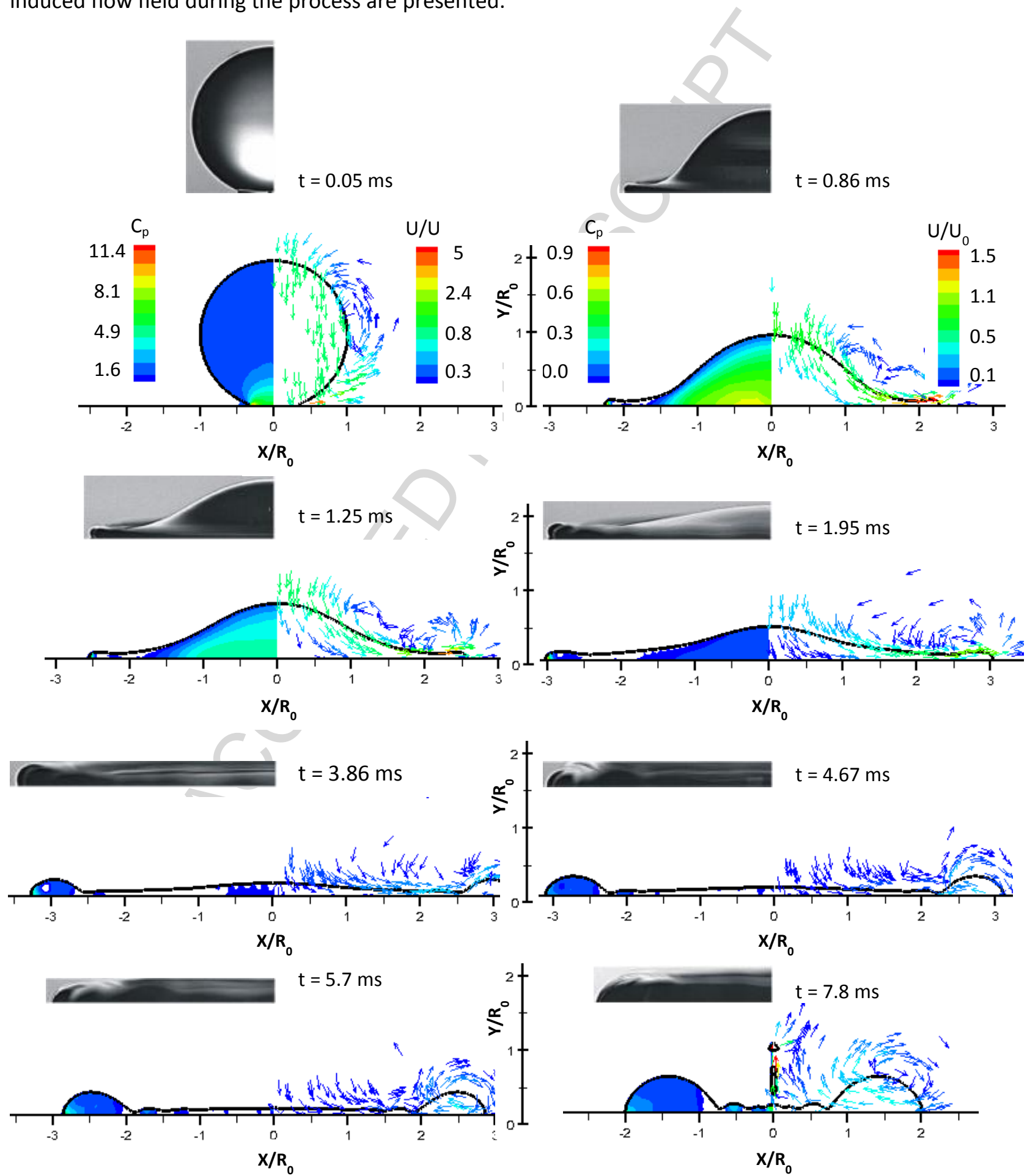

Figure 12. Comparison with experiment for Case4. HW-HCA-LH (Sikalo et al. [50]). Pictures taken from reference. Cp contour and velocity vectors colored by velocity magnitude are plotted. $C_{p}$ values below 0.01 are cutoff. The first contour legends apply to the first picture, while the second one applies to all the other. 
Typical impingement behavior is again observed in these pictures, where during the initial stage of the process, velocity magnitude reaches up to 4 times the initial impact velocity. Moreover, the vortex on the top of droplet rim is again visible, while it changes direction between $3.86 \mathrm{~ms}$ and $4.67 \mathrm{~ms}$ (receding phase). At the moment when the droplet hits the surface, $C_{p}$ can reach up to 10 times the initial kinetic energy of the droplet, while for the rest of the process the maximum one is only 0.85 of the initial kinetic energy Finally, in the last picture, at $t=7.8 \mathrm{~ms}$, the ejection of a small droplet on the symmetry axis is predicted, which comes from the quick break-up of the droplet in the symmetry axis, just as the liquid mass was retracting.

In Figure 13, the non-dimensional spreading radius is plotted against time, for all 4 cases concerning the impingement of a water droplet onto hydrophobic surfaces. It is clear that at the first stage of impingement ( $\tau=t U_{0} / D_{0}<0.2$ ) the four lines coincide in one, depicting the uniform character of the impingement process, which has been also reported by Rioboo et al. [30].

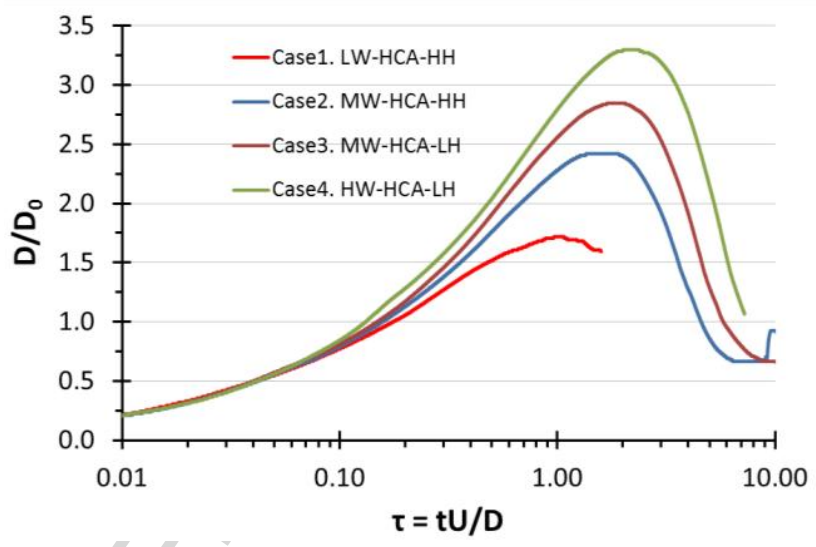

Figure 13. Non-dimensional spread factor from WFM simulation plotted against non-dimensional time for hydrophobic surfaces. $\theta_{\mathrm{adv}}=105^{\circ}-120^{\circ}$. We $=8-91, \mathrm{x}$-axis, logarithmic scale.

\subsection{Spreading on Hydrophilic surfaces}

In Figure 14, the temporal evolution of the impingement of a water droplet onto solid hydrophilic surfaces is depicted for cases 5, 7, 8 and 9. Due to the lack of numerical investigations in literature for water droplet impingement onto hydrophilic surfaces, as stated in the Introduction section, to the best of author's knowledge, the work of Fukai is the only one which was found for these 4 Cases and thus is the only one presented in Figure 14. Moreover, due to this lack of numerical investigations, the results that will be presented in this study, for all models used, concerning low and moderate Weber numbers, present an additional novel character. 
Case5. VLW-MCA-LH. We $=\mathbf{2}$

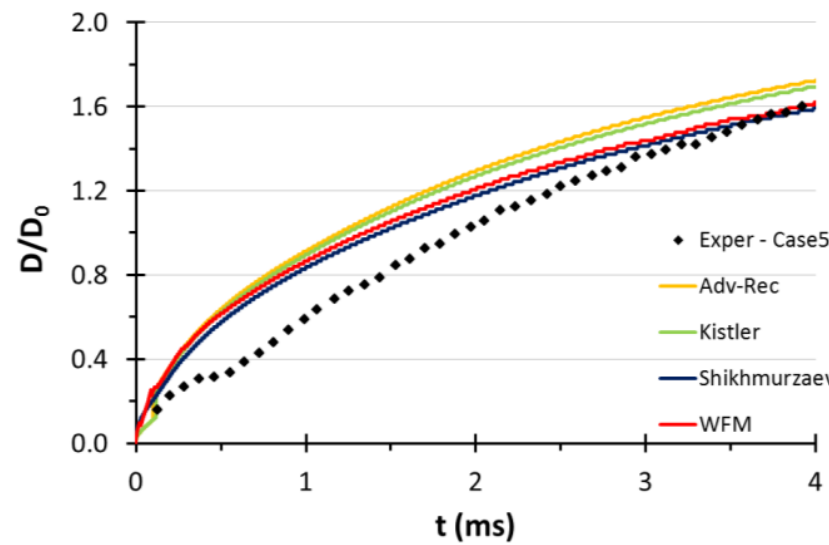

Case8. MW-LCA-LH. $\underline{\text { We }=59}$

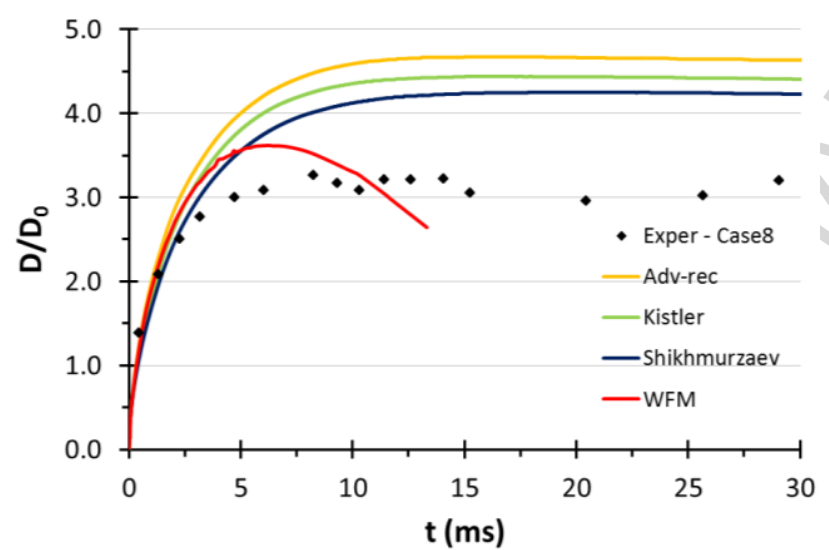

Case7. LW-MCA-LH. $\underline{\text { We }=19}$

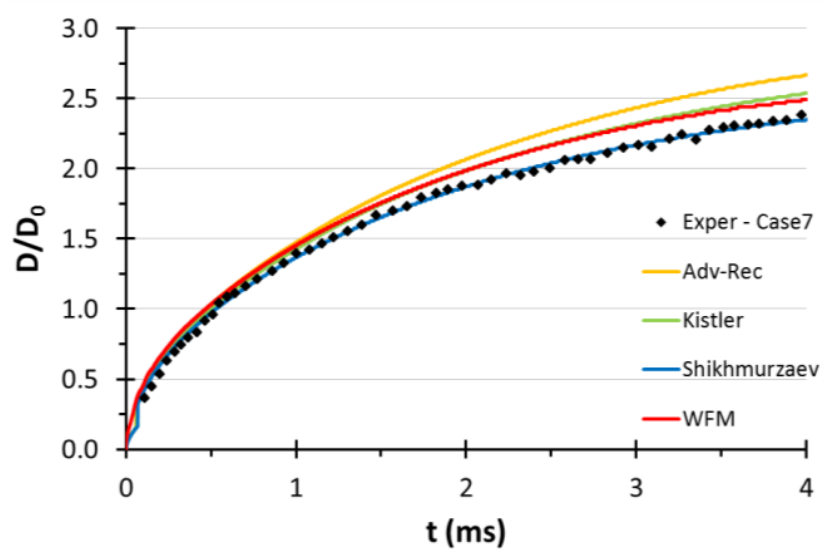

Case9. HW-MCA-HH. We $=117$

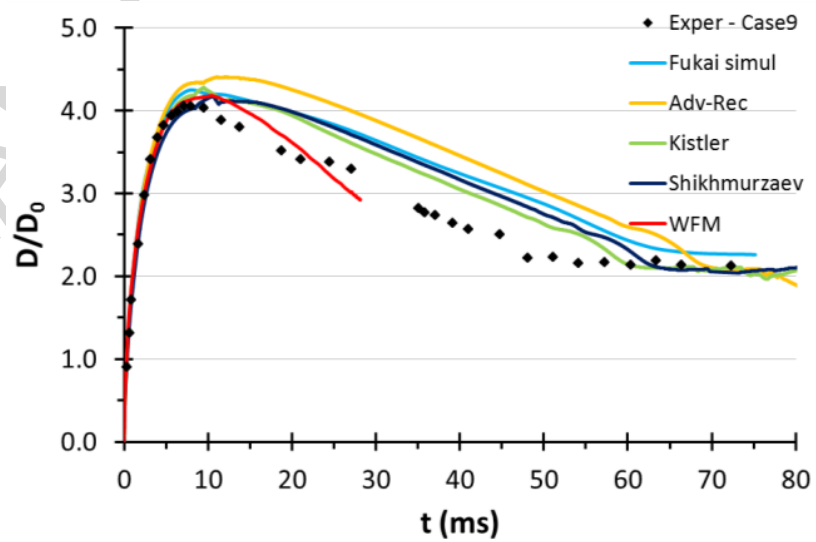

Figure 14. Comparison of WFM results against experiments and respective simulations for the hydrophilic surfaces of Cases5,7-9 listed in Table 2 (where references can be found); corresponding predictions from other dynamic contact angle models found in literature are also indicated.

The first two experimental cases presented in Figure 14 are taken from the work of Roux et al. [40] and concern the impingement of a water droplet onto a hydrophilic surface at low Weber numbers (We=2 and 19). In their work, Roux et al. [40] give experimental measurements for the time evolution of droplet radius only for the first $4 \mathrm{~ms}$ of the impingement, while they present the droplet maximum radius in one Figure relating that to the Reynolds number. Therefore, the comparison between model and experiments for these cases is limited to this specific time interval (initial $4 \mathrm{~ms}$ ), while the respective comparison with the value of maximum spreading is given in Table 2 and presented in Figure 7.

In Case5 (VLW-MCA-LH) all models overestimate droplet spreading for the initial 4ms. WFM seems to be closer to the experimental values, in comparison to the Advancing-receding model, as well as Kistler's model, in contrast to what was seen in hydrophobic surfaces. Using Shikhmurzaev's model similar results to the WFM are taken for this case. Moreover, at $4 \mathrm{~ms}$ the lines representing the time evolution of droplet spreading for all models are steeper than the experimental one, which results in an underestimation of maximum spreading, shown in Table 2. Increasing the Weber number to We=19 (Case7. LW-MCA-LH), the experimental values are approached better by the WFM simulation, at least for the initial $4 \mathrm{~ms}$ of the phenomenon. Shikhmurzaev's model gives the best results, perfectly capturing the trend of the experimental values, while all other models, as well as the WFM are quite close as well. . 
For Case8 (MW-LCA-LH), a significant over-prediction of droplet spreading is predicted by the simulation, as it is shown in Figure 14. This Case is taken from the work of Rioboo et al. [30] where it is reported that the advancing contact angle takes the value of 10 degrees (Table 2). Using this value, none of the dynamic contact angle models was able to reach a non-dimensional maximum diameter of 3.31 as measured in the experiments. The Wetting Force Model, on the other hand, predicts a non-dimensional spreading substantially lower than the other models and significantly closer to the experiments. Additionally, the recoiling of the drop is predicted, unlike to what the experiment suggests and this is the reason that the results for only the first $15 \mathrm{~ms}$ are presented.

Finally, for Case9 (MW-LCA-LH), all models seem to behave better than previously, and the maximum spread is well captured. Shikhmurzaev's dynamic contact angle model gives the best results among all the standard used contact angle models. For once more, the Wetting Force Model, seems to behave slightly better during the advancing phase, but predicts a quicker recoiling phase. In Figure 15 the prediction of "hysteresis" time for Case 9 is depicted for the WFM model in comparison to the advancing-receding model. Firstly, it is obvious that the hysteresis time lasts much longer for the Advancing-Receding model. This is shown by the "turning" of the rim at time instant $t=13.66 \mathrm{~ms}$ for the Advancing-Receding model, in conjunction with the slight "turn" in 9.93ms for the Wetting Force Model.
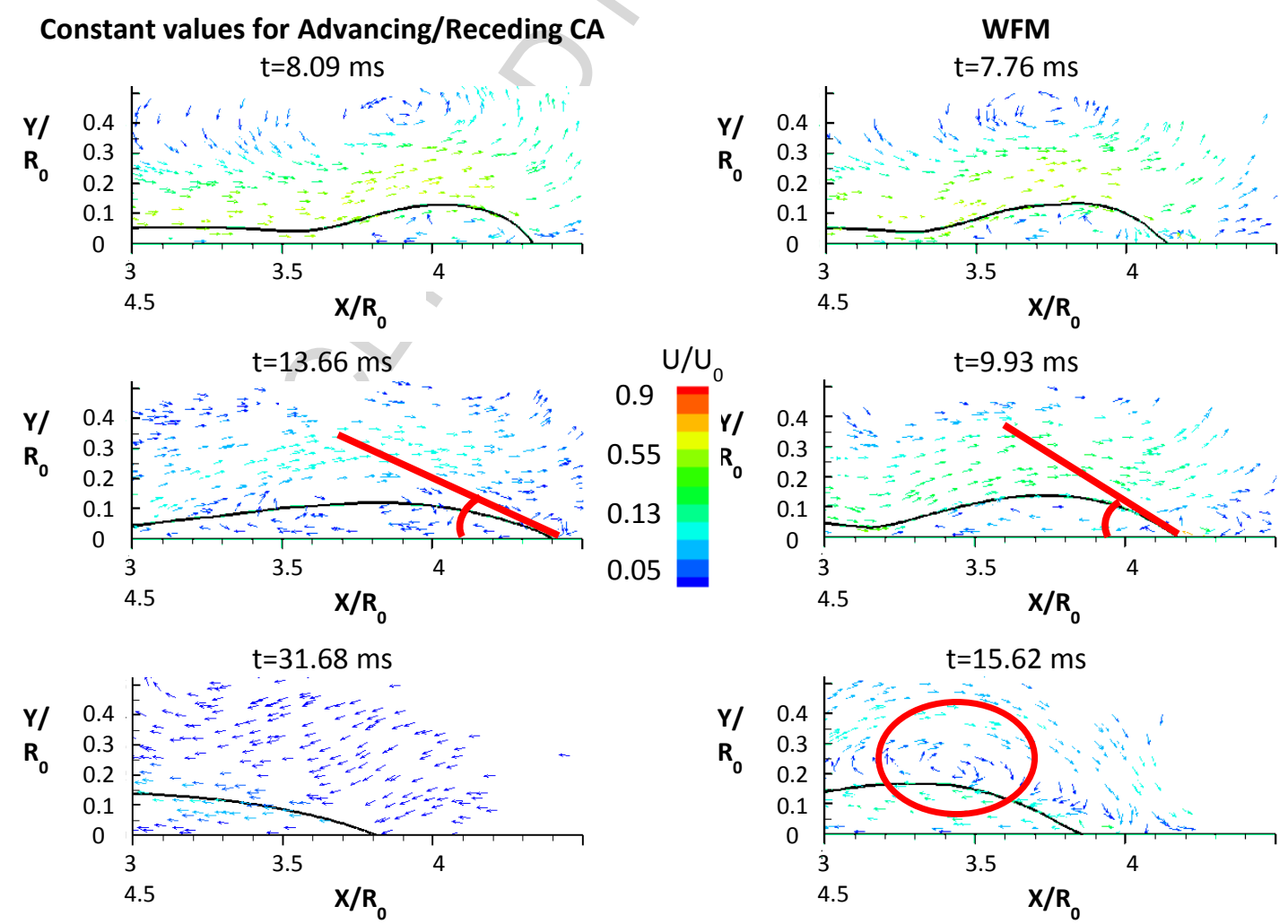

Figure 15. Prediction of "hysteresis" time for WFM in comparison to Advancing-receding model. Velocity vectors colored by velocity magnitude are plotted. Higher receding angle which results in quicker recoiling phase is predicted by the WFM.

Secondly, it is evident that the receding contact angle that is formed is not the same for the two models. The Wetting Force Model predicts a higher receding contact angle, which is the main reason for the quicker recoiling phase, as observed in Figure 14. Finally, on the third picture (15.62ms for the WFM) it is 
obvious that the highest angle that is formed using the WFM results in the prediction of a different flow field (vortex over the rim), which additionally has higher receding velocity values. Thus, a quicker receding phase is observed. The simulation stopped at $30 \mathrm{~ms}$ because droplet break-up in the symmetry axis was observed, and the present axisymmetric application of the Wetting Force Model cannot be considered as valid after that time instant. Finally, the direction turn of the vortex which lies on top of droplet rim is obvious in the transition from advancing to receding phase.

In Figure 16 and Figure 17 the shape of the drop at characteristic times during the impingement of Cases 5 (VLW-MCA-LH) and 7 (LW-MCA-LH) is presented. In Figure 16 the phenomenon is captured qualitatively.

Case5. VLW-MCA-LH - Exper (Roux et al.)

$\mathrm{We}=2$
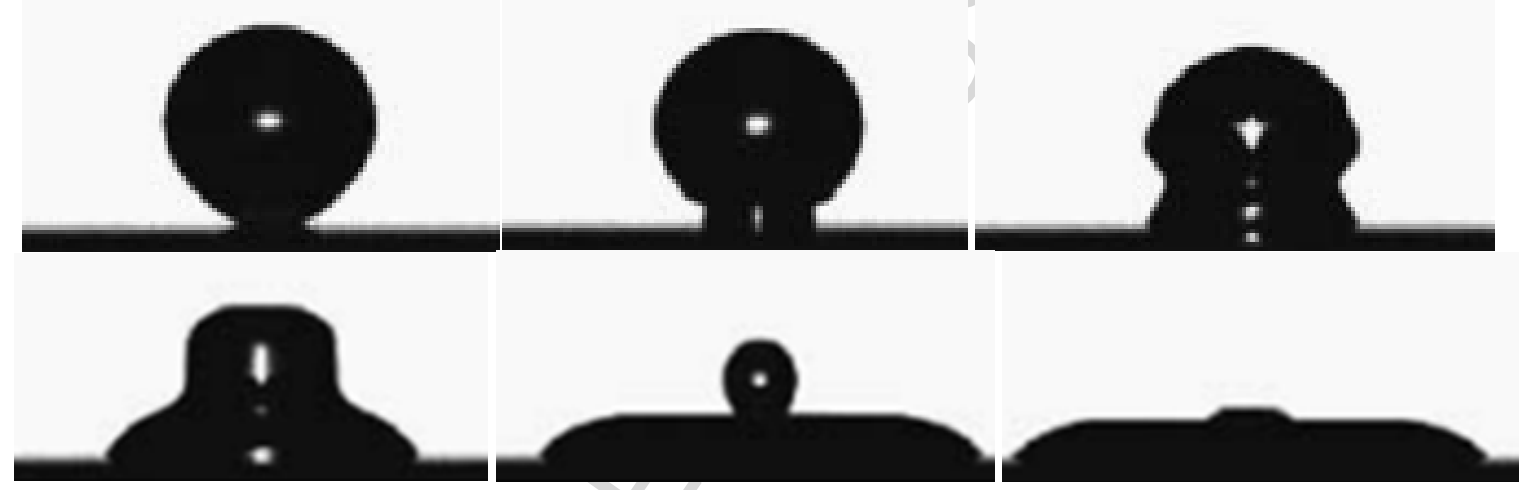

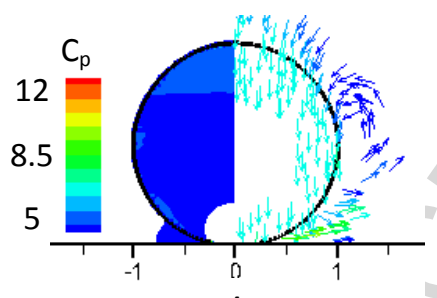

$X / R_{0}$

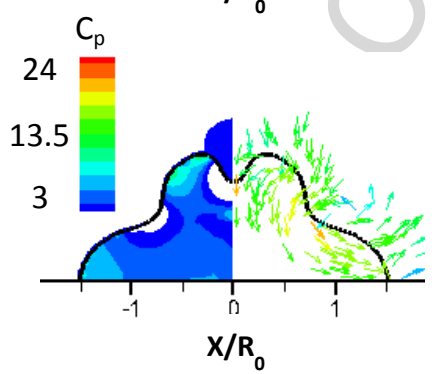

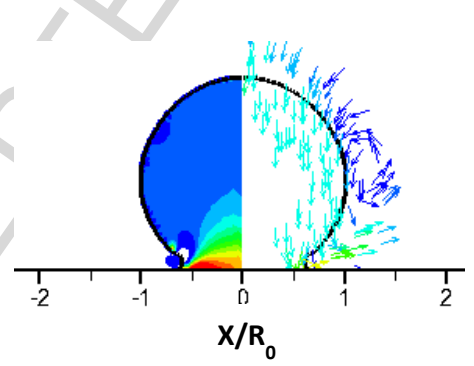

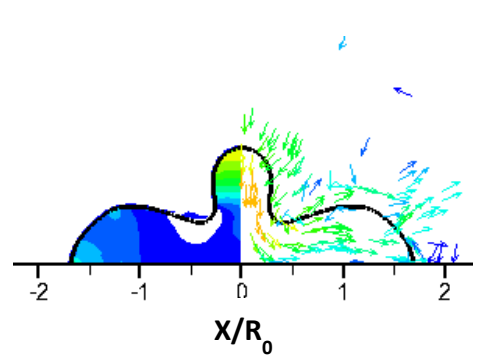

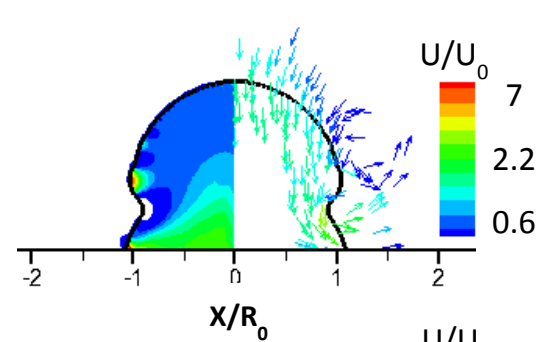

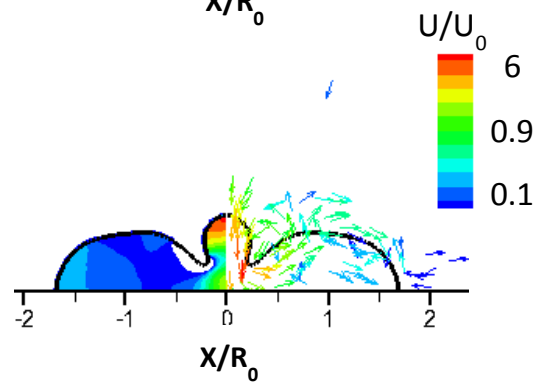

$\mathrm{t}_{\mathrm{exp}}=0.089,1.157,3.314,4.272,8.455,10.146 \mathrm{~ms}$

$t_{\text {sim }}=0.089,0.53,1.5, \quad 3.4, \quad 5.49,6.21 \mathrm{~ms}$

Figure 16. Comparison with experiment for Case5. VLW-MCA-LH. $C_{p}$ contour and velocity vectors colored by velocity magnitude are plotted. $C_{p}$ values below 0.01 are cutoff. The first contour legends apply to the first 3 pictures, while the second one applies to all the other.

However, as it was also observed in Figure 14, droplet initial spreading is overestimated, meaning that the phenomenon is predicted to be much quicker that in real time. This is evident in Figure 16, where the simulation time is much smaller than the real time. Moreover, in the first 4 experimental pictures, 
the simulated droplet shapes are very similar to the experiment, showing that the model can predict such odd shapes $(t=1.157,3.314)$ formed by the droplet at very low Weber numbers. In the next 2 pictures, a larger thickness of the liquid film is predicted, which deviates from the experiment and is the main reason for the underestimation of maximum spreading. However, as Roux et al. [40] observe, though the contact angle value at the end of the relaxation phase takes a value of approximately 15 degrees (static contact angle), the dynamic advancing contact angle formed during the advancing phase, is approximately 70 degrees. This value was used for the simulation, and as a result the dynamic contact angle was around to this value. This makes it very difficult for a simulation to capture this change in dynamic contact angle. One would expect, that using Kistler's or Shikhmurzaev's model would result in a much better representation of the phenomenon, however this was not the case (Figure 14). The pressure contours for this Case are rather peculiar. More specifically, $C_{p}$ inside the droplet is higher than expected, and has a lowest value of 3 for all pictures inside the liquid phase, instead of the typical zero value in the interface. This problem can be possibly attributed to the spurious velocities which appear in the interface region. For higher Weber number, the effect that these velocities have on the evolution of the impingement is minimal. On the other hand, at very low Weber numbers, these spurious velocities can cause an unrealistic pressure field. Additionally, because impact velocity is very low, $\mathrm{P}-\mathrm{P}_{\mathrm{oo}}$ in the $\mathrm{C}_{\mathrm{p}}$ approximation may represent the surface tension force (this is evident in very small droplets, while in this Case $D=2.4 \mathrm{~mm}$ ).

In Figure 17, the temporal evolution of impingement for Case7 (LW-MCA-LH) is compared against experimental photographs from the work of Roux et al. [40]. For this case there is both qualitative and quantitative agreement between simulation and experiment. 

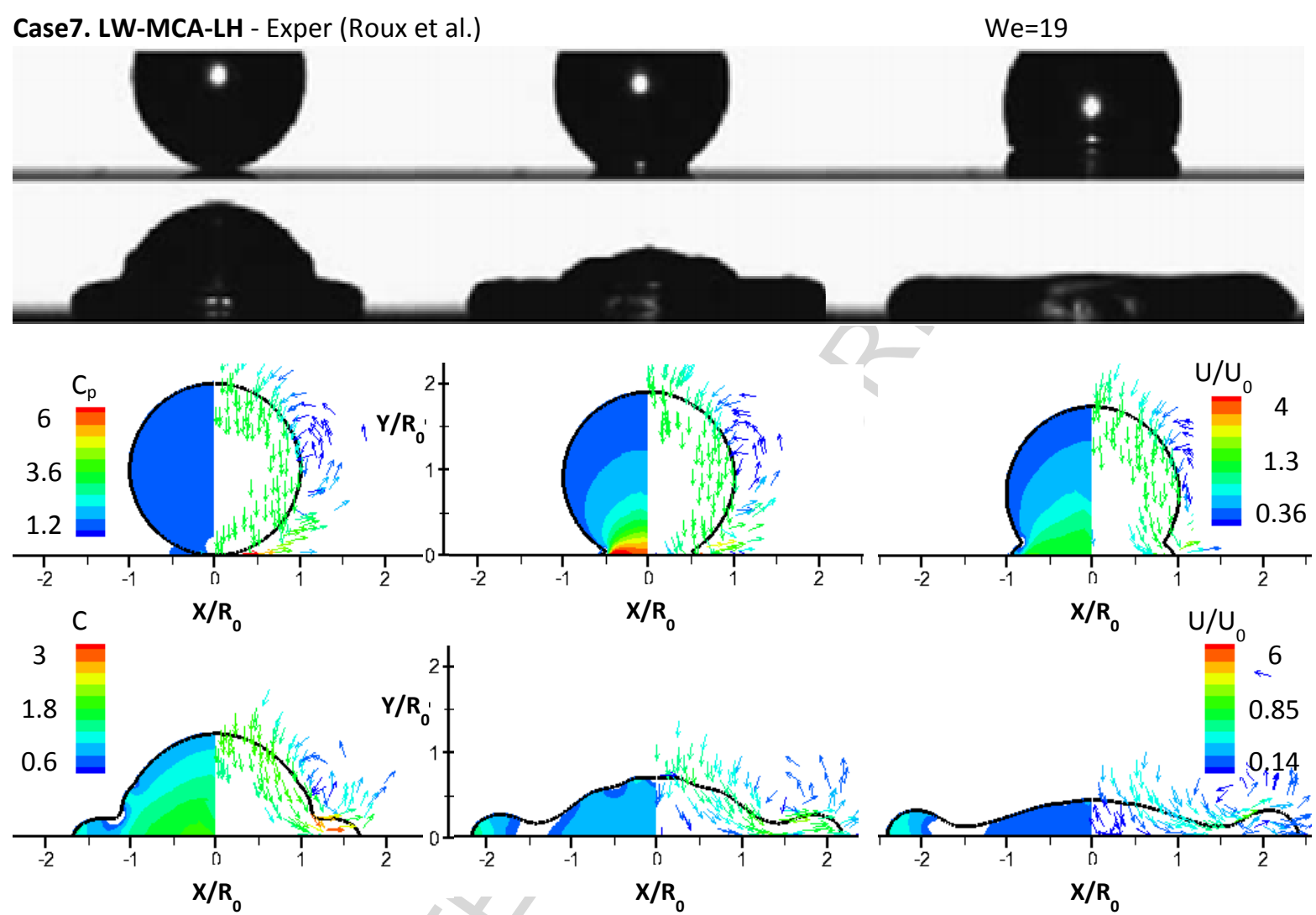

$t_{\text {exp/sim }}=0,0.132,0.484,1.452,2.508,3.608 \mathrm{~ms}$

Figure 17. Comparison with experiment for Case7 LW-MCA-LH. $C_{p}$ contour and velocity vectors colored by velocity magnitude are plotted. $C_{p}$ values below 0.01 are cutoff. The first contour legends apply to the first 3 pictures, while the second one applies to all the other.

A general observation is that WFM, in contrast to hydrophobic surfaces (Figure 10) gives results, which are closer to the experimental values than the Advancing-Receding or Kistler's model, while a quicker recoiling phase is predicted for Cases8 (MW-LCA-LH) - 9 (HW-MCA-HH). As for the other models, Shikhmurzaev's model for dynamic contact angle behaves better in all cases presented, while Kistler's model for hydrophilic surfaces exhibits significant improvement in comparison to the advancingreceding model. In hydrophobic surfaces the results for these models were similar. In Figure 18, the nondimensional droplet diameter is plotted against time, which is non-dimensionalised by $\mathrm{U}_{0}{ }^{-1 / 2}$, a factor proposed by Antonini et al. [38] for a uniform representation of droplet spreading radius on hydrophilic surfaces. Again, all lines coincide in one for the initial stage of impingement, while afterwards the effect of inertia plays significant role. The values of $\mathrm{x}$-axis do not correspond to a real quantity. 


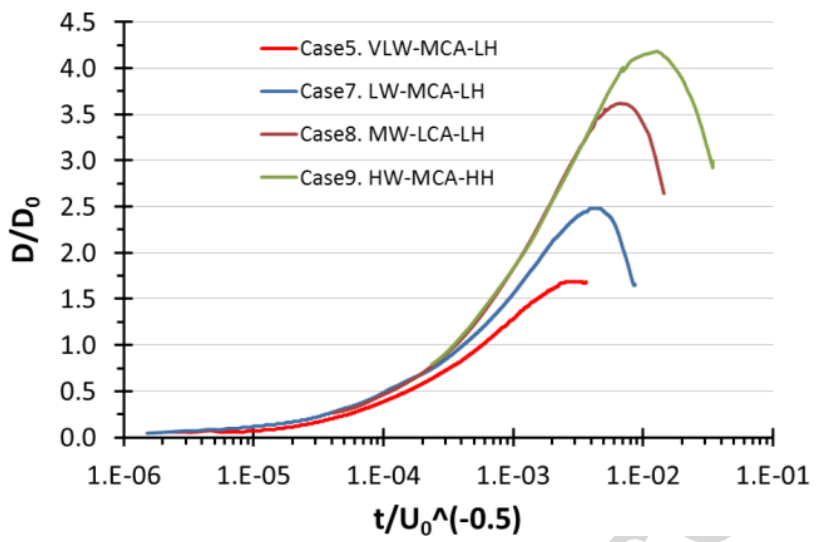

Figure 18. Non-dimensional spread factor from WFM simulation plotted against non-dimensional time for hydrophilic surfaces. $\theta_{\mathrm{adv}}=10^{\circ}-70^{\circ}$. We $=0.2-117, \mathrm{x}$-axis, logarithmic scale.

\subsection{Impact at very low Weber numbers}

Due to the significant under prediction of the maximum spread in Case5 (VLW-MCA-LH) for low Weber number $(\mathrm{We}=2)$ it was deemed necessary that another Case should be run for justification reasons. This case (Case6 VLW-LCA-LH in Table 2), for an extremely low Weber number (We=0.2) was taken from the experimental work of Park et al. [32], who do not present photographs, but only the time evolution of droplet radius. In Figure 19, results of all models used in this study are presented both for Case5 (VLWMCA-LH) and Case6 (VLW-LCA-LH). During the initial 4 ms of the phenomenon, simulation results on one case (Case5 VLW-MCA-LH) overestimate droplet initial spreading, while on the other (Case6 VLW-LCA-LH) underestimate it. This clearly shows how difficult from a numerical point of view, the simulation at low Weber numbers is, and how sensitive to all conditions of the solid surface the real phenomenon is, always taking into account the uncertainty of experimental measurements. Concerning the whole phenomenon $(0<\mathrm{t}<60 \mathrm{~ms}$ ), it is observed that the model cannot predict a slow deposition as seen during the experiments for Case6 (VLW-LCA-LH) (Figure 19). More specifically, the droplet, after it reaches its maximum radius, oscillates until it comes to rest, unlike to what the experiment (slow deposition) depicts. This behavior is also evident in Case5 (VLW-MCA-LH); however the temporal evolution of $D_{\max } / D_{0}$ is not available in order to compare it. This means that the dissipation of initial kinetic energy is not well predicted by the model. 
Case5. VLW-MCA-LH , We=2

$\mathrm{t}<4 \mathrm{~ms}$

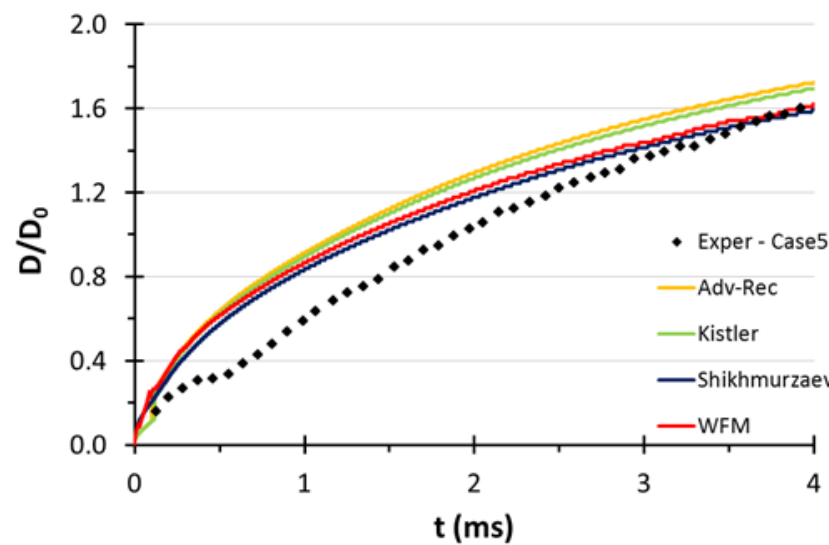

$0<\mathrm{t}<60 \mathrm{~ms}$

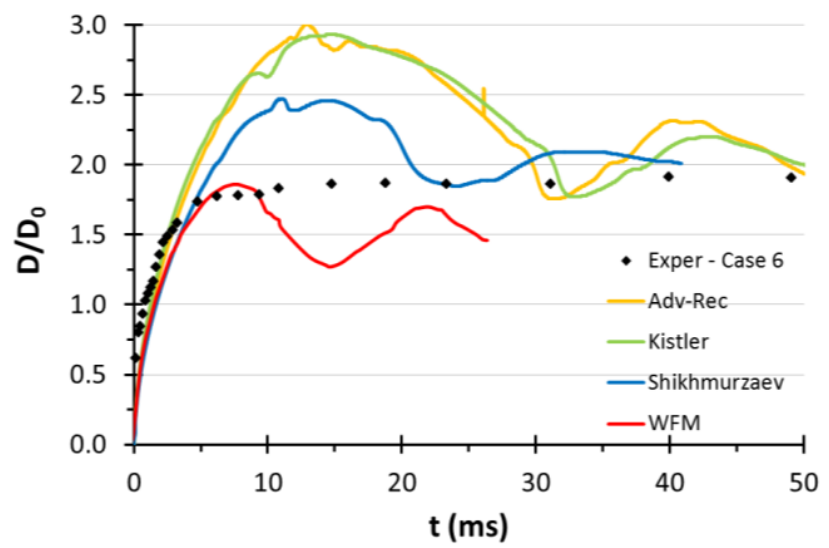

Case6. VLW-LCA-LH , We $=0.2$
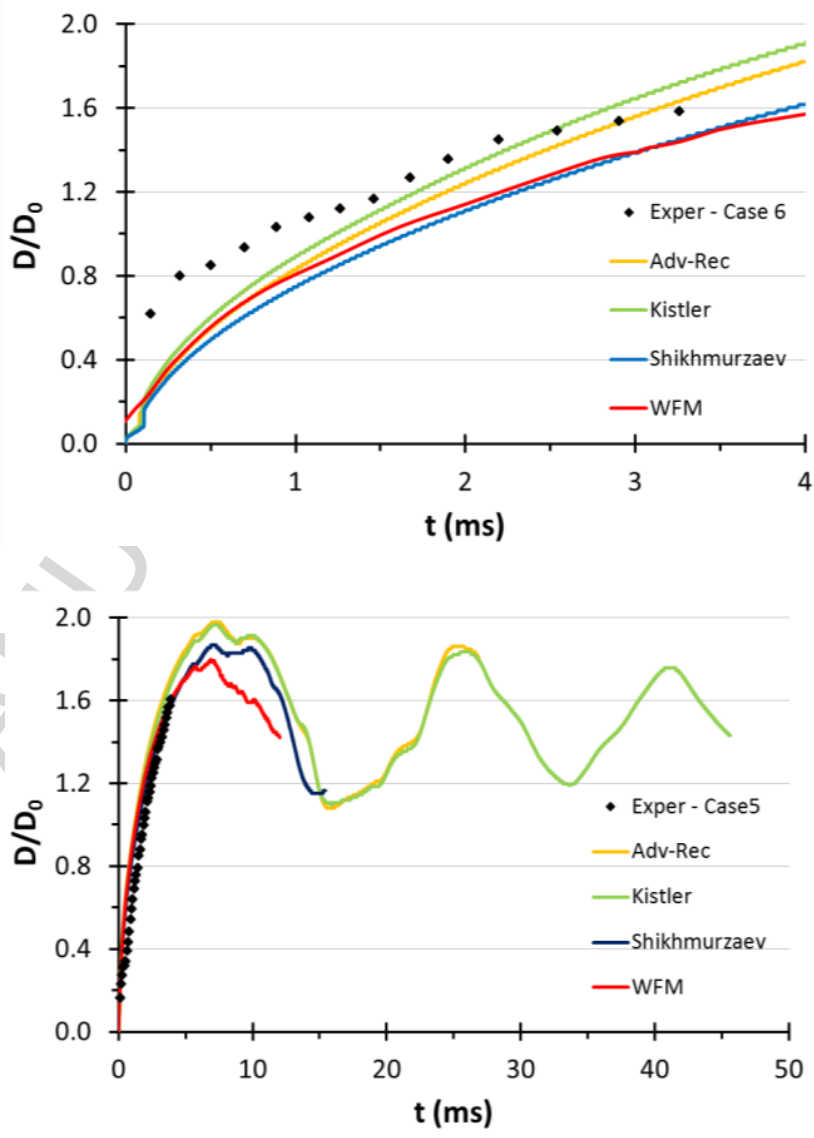

Figure 19. Comparison of simulated results for very low Weber numbers.

\section{Conclusions}

A new numerical model, the Wetting Force Model, for modelling the capillary adhesion forces acting at the three-phase contact line of a liquid drop impacting onto a solid surface was for the first time presented and applied to numerical simulations. The proposed approach orients to the detailed description of low and medium We number impacts, for which capillary forces can be considered to be influential in terms of the spreading dynamics, while the novelty of the new model, compared to most of the models presented in the literature, is the use of a completely different approach with respect to the contact angle calculation computation. Indeed, the dynamic contact angle does not have to be provided as a boundary conditions of the wall, i.e. as an input for the simulation, but is derived as a result of the acting wetting forces at the droplet rim under dynamic conditions. The model assumes that an adhesion force, due to capillary interaction between the liquid and the solid surface, acts at the contact line. This force comes into play when the dynamic contact angle differs from the equilibrium contact angle, which is the advancing contact angle during spreading and the receding contact angle during recoiling. The implementation of this force was achieved by its addition as an extra stress term in the Navier-Stokes momentum equation. The derived numerical results were compared not only against experimental data, but also against the corresponding simulation results of other researchers and three different contact 
angle models (namely Advancing-Receding, Kistler's and Shikhmurzaev's dynamic contact angle models). For all the cases, good agreement with experimental data is observed, with a slight over-prediction $(<15 \%)$ of droplet maximum spreading. The comparison with all other simulation models delivers similar results, with the exception of the dynamic contact angle model of Shikhmurzaev, which in most cases gives the best results in terms of spreading ratios. In particular, although the recoiling phase, which was observed in hydrophilic surfaces at moderate and high Weber numbers, is quicker than the experimentally observed, on hydrophilic surfaces and for moderate Weber numbers, the improvement in droplet maximum spreading owed to the use of WFM in comparison to Shikhmurzaev's model is very promising.

\section{Acknowledgements}

The present work was funded by the Marie Curie Fellowship (FP7-PEOPLE-2012-IEF) with Grant Agreement number 329500 funded by the European Commission entitled as "Non Flat ImpingementDroplet Impingement on Non-flat Surfaces". CA acknowledges funding through a Marie Curie IntraEuropean Fellowship (ICE $\left.{ }^{2}, 301174\right)$. The authors would like to thank Mr. Sergios Goudanis for his kind help with the 3D representation of the results using the commercial package LOOK CFD (www.lookcfd.com)

\section{References}

1. Kim, J., Spray cooling heat transfer: The state of the art. International Journal of Heat and Fluid Flow, 2007. 28(4): p. 753-767.

2. Kandlikar, S.G. and A.V. Bapat, Evaluation of Jet Impingement, Spray and Microchannel Chip Cooling Options for High Heat Flux Removal. Heat Transfer Engineering, 2007. 28(11): p. 911-923.

3. Han, Z., Z. Xu, and N. Trigui, Spray/wall interaction models for multidimensional engine simulation. International Journal of Engine Research, 2000. 1(1): p. 127-146.

4. Zhao, F., M.C. Lai, and D.L. Harrington, Automotive spark-ignited direct-injection gasoline engines. Progress in Energy and Combustion Science, 1999. 25(5): p. 437-562.

5. Pasandideh-Fard, M., et al., Splat shapes in a thermal spray coating process: Simulations and experiments. Journal of Thermal Spray Technology, 2002. 11(2): p. 206-217.

6. Addy, H.E., Ice accretions and icing effects for modern airfoils [microform] / Harold E. Addy, Jr. NASA technical paper ; 210031., ed. N.G.R. Center2000, [Cleveland, Ohio] : [Springfield, Va: National Aeronautics and Space Administration, Glenn Research Center ; National Technical Information Service, distributor.

7. Antonini, C., et al., Drop Rebound after Impact: The Role of the Receding Contact Angle. Langmuir, 2013.

8. Faustini, M., et al., Hydrophobic, Antireflective, Self-Cleaning, and Antifogging Sol-Gel Coatings: An Example of Multifunctional Nanostructured Materials for Photovoltaic Cells. Chemistry of Materials, 2010. 22(15): p. 4406-4413.

9. Young, T., An Essay on the Cohesion of Fluids. Philosophical Transactions of the Royal Society of London, 1805. 95: p. 65-87.

10. Worthington, A.M., On the Forms Assumed by Drops of Liquids Falling Vertically on a Horizontal Plate. Proceedings of the Royal Society of London, 1876. 25(171-178): p. 261-272. 
11. Rein, M., Phenomena of liquid drop impact on solid and liquid surfaces. Fluid Dynamics Research, 1993. 12(2): p. 61-93.

12. Yarin, A.L., DROP IMPACT DYNAMICS: Splashing, Spreading, Receding, Bouncing. Annual Review of Fluid Mechanics, 2006. 38(1): p. 159-192.

13. Marengo, M., et al., Drop collisions with simple and complex surfaces. Current Opinion in Colloid \& Interface Science, 2011. 16(4): p. 292-302.

14. Dussan, E.B., On the Spreading of Liquids on Solid Surfaces: Static and Dynamic Contact Lines. Annual Review of Fluid Mechanics, 1979. 11(1): p. 371-400.

15. Gennes, P.G.D., Wetting: statics and dynamics. Reviews of Modern Physics, 1985. 57(3): p. 827863.

16. Shikhmurzaev, Y.D., Singularities at the moving contact line. Mathematical, physical and computational aspects. Physica D: Nonlinear Phenomena, 2006. 217(2): p. 121-133.

17. Chandra, S. and C.T. Avedisian, On the Collision of a Droplet with a Solid Surface. Proceedings of the Royal Society of London. Series A: Mathematical and Physical Sciences, 1991. 432(1884): p. 13-41.

18. Pasandideh-Fard, M., et al., Capillary effects during droplet impact on a solid surface. Physics of Fluids, 1996. 8(3): p. 650-659.

19. Mao, T., D.C.S. Kuhn, and H. Tran, Spread and rebound of liquid droplets upon impact on flat surfaces. AIChE Journal, 1997. 43(9): p. 2169-2179.

20. Ukiwe, C. and D.Y. Kwok, On the Maximum Spreading Diameter of Impacting Droplets on WellPrepared Solid Surfaces. Langmuir, 2004. 21(2): p. 666-673.

21. Vadillo, D.C., et al., Dynamic contact angle effects onto the maximum drop impact spreading on solid surfaces. Physics of Fluids, 2009. 21(12): p. 122002-8.

22. Kim, H.Y. and J.H. Chun, The recoiling of liquid droplets upon collision with solid surfaces. Physics of Fluids, 2001. 13(3): p. 643-659.

23. An , S.M. and S.Y. Lee, One-dimensional model for the prediction of impact dynamics of a shearthinning liquid drop on dry solid surfaces. Atomization and Sprays, 2012. 22(5): p. 371-389.

24. Scheller, B.L. and D.W. Bousfield, Newtonian drop impact with a solid surface. AlChE Journal, 1995. 41(6): p. 1357-1367.

25. Zhang, X. and O.A. Basaran, Dynamic Surface Tension Effects in Impact of a Drop with a Solid Surface. Journal of colloid and interface science, 1997. 187(1): p. 166-178.

26. Range, K. and F. Feuillebois, Influence of Surface Roughness on Liquid Drop Impact. Journal of colloid and interface science, 1998. 203(1): p. 16-30.

27. Crooks, R., J. Cooper-White, and D.V. Boger, The role of dynamic surface tension and elasticity on the dynamics of drop impact. Chemical Engineering Science, 2001. 56(19): p. 5575-5592.

28. Rioboo, R., C. Tropea, and M. Marengo, Outcomes from a drop impact on solid surfaces. Atomization and Sprays, 2001. 11(2): p. 12.

29. Richard, D., C. Clanet, and D. Quéré, Surface phenomena: Contact time of a bouncing drop. Nature, 2002. 417(6891): p. 811-811.

30. Rioboo, R., M. Marengo, and C. Tropea, Time evolution of liquid drop impact onto solid, dry surfaces. Experiments in Fluids, 2002. 33(1): p. 112-124.

31. Sikalo, S., et al., Analysis of impact of droplets on horizontal surfaces. Experimental Thermal and Fluid Science, 2002. 25(7): p. 503-510.

32. Park, H., et al., Single drop impaction on a solid surface. AIChE Journal, 2003. 49(10): p. 24612471.

33. Clanet, C., et al., Maximal deformation of an impacting drop. Journal of Fluid Mechanics, 2004. 517: p. 199-208. 
34. Roux, D.C.D. and J.J. Cooper-White, Dynamics of water spreading on a glass surface. Journal of colloid and interface science, 2004. 277(2): p. 424-436.

35. Bartolo, D., C. Josserand, and D. Bonn, Retraction dynamics of aqueous drops upon impact on non-wetting surfaces. Journal of Fluid Mechanics, 2005. 545: p. 329-338.

36. Bayer, I.S. and C.M. Megaridis, Contact angle dynamics in droplets impacting on flat surfaces with different wetting characteristics. Journal of Fluid Mechanics, 2006. 558: p. 415-449.

37. Hung, Y.-L., et al., Initial wetting velocity of droplet impact and spreading: Water on glass and parafilm. Colloids and Surfaces A: Physicochemical and Engineering Aspects, 2011. 384(1-3): p. 172-179.

38. Antonini, C., A. Amirfazli, and M. Marengo, Drop impact and wettability: From hydrophilic to superhydrophobic surfaces. Physics of Fluids, 2012. 24(10): p. 102104-13.

39. Jiang, T.-S., O.H. Soo-Gun, and J.C. Slattery, Correlation for dynamic contact angle. Journal of colloid and interface science, 1979. 69(1): p. 74-77.

40. Roux, D.C. and J.J. Cooper-White, Dynamics of water spreading on a glass surface. Journal of colloid and interface science, 2004. 277(2): p. 424-436.

41. Roisman, I.V., et al., Drop impact onto a dry surface: Role of the dynamic contact angle. Colloids and Surfaces A: Physicochemical and Engineering Aspects, 2008. 322(1-3): p. 183-191.

42. Harlow, F.H. and J.E. Welch, Numerical Calculation of Time-Dependent Viscous Incompressible Flow of Fluid with Free Surface. Physics of Fluids, 1965. 8(12): p. 2182-2189.

43. Fukai, J., et al., Wetting effects on the spreading of a liquid droplet colliding with a flat surface: Experiment and modeling. Physics of Fluids, 1995. 7(2): p. 236-247.

44. Zhao, Z., D. Poulikakos, and J. Fukai, Heat transfer and fluid dynamics during the collision of a liquid droplet on a substrate - I. Modeling. International Journal of Heat and Mass Transfer, 1996. 39(13): p. 2771-2789.

45. Manservisi, S. and R. Scardovelli, A variational approach to the contact angle dynamics of spreading droplets. Computers \& Fluids, 2009. 38(2): p. 406-424.

46. Muradoglu, M. and S. Tasoglu, A front-tracking method for computational modeling of impact and spreading of viscous droplets on solid walls. Computers \& Fluids, 2010. 39(4): p. 615-625.

47. Shin, S. and D. Juric, Simulation of droplet impact on a solid surface using the level contour reconstruction method. Journal of Mechanical Science and Technology, 2009. 23(9): p. 24342443.

48. Pasandideh-Fard, M., et al., Deposition of tin droplets on a steel plate: simulations and experiments. International Journal of Heat and Mass Transfer, 1998. 41(19): p. 2929-2945.

49. Pasandideh-Fard, M., et al., Cooling effectiveness of a water drop impinging on a hot surface. International Journal of Heat and Fluid Flow, 2001. 22(2): p. 201-210.

50. Sikalo, S., et al., Dynamic contact angle of spreading droplets: Experiments and simulations. Physics of Fluids, 2005. 17(6): p. 062103-13.

51. Lunkad, S.F., V.V. Buwa, and K.D.P. Nigam, Numerical simulations of drop impact and spreading on horizontal and inclined surfaces. Chemical Engineering Science, 2007. 62(24): p. 7214-7224.

52. Strotos, G., et al., Non-dimensionalisation parameters for predicting the cooling effectiveness of droplets impinging on moderate temperature solid surfaces. International Journal of Thermal Sciences, 2011. 50(5): p. 698-711.

53. Nikolopoulos, N., A. Theodorakakos, and G. Bergeles, A numerical investigation of the evaporation process of a liquid droplet impinging onto a hot substrate. International Journal of Heat and Mass Transfer, 2007. 50(1-2): p. 303-319.

54. Yokoi, K., et al., Numerical studies of the influence of the dynamic contact angle on a droplet impacting on a dry surface. Physics of Fluids, 2009. 21(7): p. 072102-12. 
55. Caviezel, D., C. Narayanan, and D. Lakehal, Adherence and bouncing of liquid droplets impacting on dry surfaces. Microfluidics and Nanofluidics, 2008. 5(4): p. 469-478.

56. Griebel, M. and M. Klitz, Simulation of Droplet Impact with Dynamic Contact Angle Boundary Conditions. INS Preprint No. 1302, 2013.

57. Gunjal, P.R., V.V. Ranade, and R.V. Chaudhari, Dynamics of drop impact on solid surface: Experiments and VOF simulations. AIChE Journal, 2005. 51(1): p. 59-78.

58. Bussmann, M., S. Chandra, and J. Mostaghimi, Modeling the splash of a droplet impacting a solid surface. Physics of Fluids, 2000. 12(12): p. 3121-3132.

59. Francois, M. and W. Shyy, Computations of drop dynamics with the immersed boundary method, Part 2: Drop impact and heat transfer. Numerical Heat Transfer, Part B: Fundamentals, 2003. 44(2): p. 119-143.

60. Ganesan, S., On the dynamic contact angle in simulation of impinging droplets with sharp interface methods. Microfluidics and Nanofluidics, 2013. 14(3-4): p. 615-625.

61. Mukherjee, S. and J. Abraham, Investigations of drop impact on dry walls with a latticeBoltzmann model. Journal of colloid and interface science, 2007. 312(2): p. 341-354.

62. Tanaka, Y., et al., Numerical simulation of dynamic behavior of droplet on solid surface by the two-phase lattice Boltzmann method. Computers \& Fluids, 2011. 40(1): p. 68-78.

63. Dong, S., On imposing dynamic contact-angle boundary conditions for wall-bounded liquid-gas flows. Computer Methods in Applied Mechanics and Engineering, 2012. 247-248(0): p. 179-200.

64. Kamnis, S. and S. Gu, Numerical modelling of droplet impingement. Journal of Physics D: Applied Physics, 2005. 38(19): p. 3664.

65. Strotos, G., et al., Numerical investigation of the cooling effectiveness of a droplet impinging on a heated surface. International Journal of Heat and Mass Transfer, 2008. 51(19-20): p. 47284742.

66. Strotos, G., et al., Numerical investigation on the evaporation of droplets depositing on heated surfaces at low Weber numbers. International Journal of Heat and Mass Transfer, 2008. 51(7-8): p. 1516-1529.

67. Strotos, G., et al., Cooling effectiveness of droplets at low Weber numbers: Effect of temperature. International Journal of Thermal Sciences, 2013. 72(0): p. 60-72.

68. Sui, Y., H. Ding, and P.D.M. Spelt, Numerical Simulations of Flows with Moving Contact Lines. Annual Review of Fluid Mechanics, 2014. 46(1): p. 97-119.

69. Brackbill, J.U., D.B. Kothe, and C. Zemach, A continuum method for modeling surface tension. Journal of Computational Physics, 1992. 100(2): p. 335-354.

70. Ashish Saha, A. and S.K. Mitra, Effect of dynamic contact angle in a volume of fluid (VOF) model for a microfluidic capillary flow. Journal of colloid and interface science, 2009. 339(2): p. 461-480.

71. Olsson, E. and G. Kreiss, A conservative level set method for two phase flow. Journal of Computational Physics, 2005. 210(1): p. 225-246.

72. Kistler, S.F., Hydrodynamics of wetting. Wettability, 1993. 311.

73. Shikhmurzaev, Y.D., Capillary flows with forming interfaces2007: CRC Press.

74. Šikalo, Š., C. Tropea, and E.N. Ganić, Impact of droplets onto inclined surfaces. Journal of colloid and interface science, 2005. 286(2): p. 661-669.

75. Antonini, C., et al., General Methodology for Evaluating the Adhesion Force of Drops and Bubbles on Solid Surfaces. Langmuir, 2009. 25(11): p. 6143-6154.

76. Extrand, C.W. and Y. Kumagai, Liquid Drops on an Inclined Plane: The Relation between Contact Angles, Drop Shape, and Retentive Force. Journal of colloid and interface science, 1995. 170(2): p. 515-521.

77. Hirt, C.W. and B.D. Nichols, Volume of fluid (VOF) method for the dynamics of free boundaries. Journal of Computational Physics, 1981. 39(1): p. 201-225. 
78. Nikolopoulos, N., A. Theodorakakos, and G. Bergeles, Normal impingement of a droplet onto a wall film: a numerical investigation. International Journal of Heat and Fluid Flow, 2005. 26(1): p. 119-132.

79. FLUENT 14.5. Theory Guide, 2011.

80. Aliabadi, S. and T.E. Tezduyar, Stabilized-finite-element/interface-capturing technique for parallel computation of unsteady flows with interfaces. Computer Methods in Applied Mechanics and Engineering, 2000. 190(3-4): p. 243-261.

81. Olsson, E., G. Kreiss, and S. Zahedi, A conservative level set method for two phase flow II. Journal of Computational Physics, 2007. 225(1): p. 785-807.

82. So, K.K., X.Y. Hu, and N.A. Adams, Anti-diffusion method for interface steepening in two-phase incompressible flow. Journal of Computational Physics, 2011. 230(13): p. 5155-5177.

83. Boger, M., et al., Reduction of parasitic currents in the DNS VOF code FS3D. 12th Workshop on Two-Phase Flow Predictions, 2010.

84. Ubbink, O., Numerical prediction of two fluid systems with sharp interfaces, 1997, Imperial College.

85. Nikolopoulos, N., K.S. Nikas, and G. Bergeles, A numerical investigation of central binary collision of droplets. Computers \& Fluids, 2009. 38(6): p. 1191-1202.

86. Nikolopoulos, N., A. Theodorakakos, and G. Bergeles, Three-dimensional numerical investigation of a droplet impinging normally onto a wall film. Journal of Computational Physics, 2007. 225(1): p. 322-341.

87. Varagnolo, S., et al., Stick-Slip Sliding of Water Drops on Chemically Heterogeneous Surfaces. Physical Review Letters, 2013. 111(6): p. 066101.

88. Theodorakakos, A. and G. Bergeles, Simulation of sharp gas-liquid interface using VOF method and adaptive grid local refinement around the interface. International Journal for Numerical Methods in Fluids, 2004. 45(4): p. 421-439.

89. Killion, J.D. and S. Garimella, Simulation of Pendant Droplets and Falling Films in Horizontal Tube Absorbers. Journal of Heat Transfer, 2005. 126(6): p. 1003-1013.

90. Maitra, T., et al., On the Nanoengineering of Superhydrophobic and Impalement Resistant Surface Textures below the Freezing Temperature. Nano Letters, 2013. 

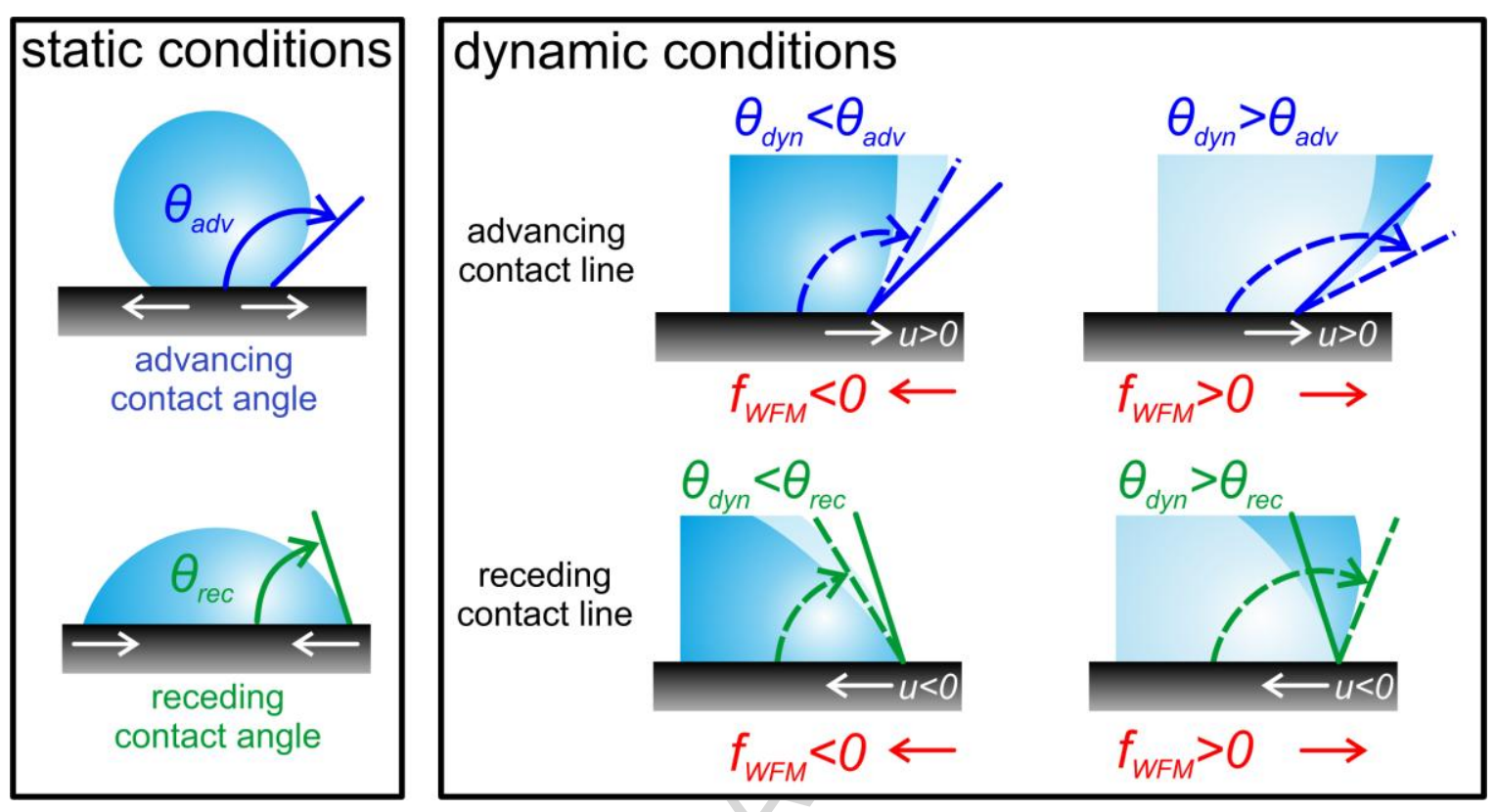

Graphical abstract 


\section{Highlights:}

1. Numerical prediction of low viscous droplet dynamics after impact on a solid substrate.

2. The inclusion of an adhesion force at the contact line of the droplet is proposed.

3. Dynamic contact angle is derived from simulation and not set as a boundary condition.

4. Good agreement with experiments for a wide range of Weber numbers is achieved.

5. Good agreement with experiments for hydrophobic and hydrophilic surfaces is achieved. 